Review Article

\title{
Optical Coherence Tomography Imaging of the Lamina Cribrosa: Structural Biomarkers in Nonglaucomatous Diseases
}

\author{
Alice Paulo, ${ }^{1}$ Pedro G. Vaz $\mathbb{D}^{1},{ }^{1}$ Danilo Andrade De Jesus $\mathbb{D},{ }^{2}$ Luisa Sánchez Brea $\left(\mathbb{D},{ }^{2}\right.$

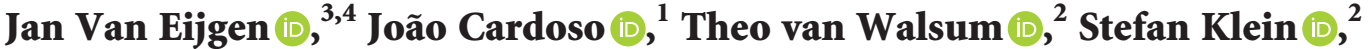 \\ Ingeborg Stalmans $₫{ }^{3,4}$ and João Barbosa Breda $\oplus^{3,5,6}$ \\ ${ }^{1}$ Laboratory for Instrumentation, Biomedical Engineering and Radiation Physics (LIBPhys-UC), Department of Physics, \\ University of Coimbra, Coimbra, Portugal \\ ${ }^{2}$ Biomedical Imaging Group Rotterdam, Department of Radiology \& Nuclear Medicine, Erasmus MC, Rotterdam, Netherlands \\ ${ }^{3}$ Research Group Ophthalmology, Department of Neurosciences, KU Leuven, Leuven, Belgium \\ ${ }^{4}$ Department of Ophthalmology, University Hospitals UZ Leuven, Leuven, Belgium \\ ${ }^{5}$ Cardiovascular ReD Center, Faculty of Medicine of the University of Porto, Porto, Portugal \\ ${ }^{6}$ Ophthalmology Department, Centro Hospitalar e Universitário São João, Porto, Portugal
}

Correspondence should be addressed to Danilo Andrade De Jesus; d.andradedejesus@erasmusmc.nl

Received 14 September 2020; Revised 28 January 2021; Accepted 5 February 2021; Published 19 February 2021

Academic Editor: Carlo Lavia

Copyright (c) 2021 Alice Paulo et al. This is an open access article distributed under the Creative Commons Attribution License, which permits unrestricted use, distribution, and reproduction in any medium, provided the original work is properly cited.

\begin{abstract}
The lamina cribrosa (LC) is an active structure that responds to the strain by changing its morphology. Abnormal changes in LC morphology are usually associated with, and indicative of, certain pathologies such as glaucoma, intraocular hypertension, and myopia. Recent developments in optical coherence tomography (OCT) have enabled detailed in vivo studies about the architectural characteristics of the LC. Structural characteristics of the LC have been widely explored in glaucoma management. However, information about which LC biomarkers could be useful for the diagnosis, and follow-up, of other diseases besides glaucoma is scarce. Hence, this literature review aims to summarize the role of the LC in nonophthalmic and ophthalmic diseases other than glaucoma. PubMed was used to perform a systematic review on the LC features that can be extracted from OCT images. All imaging features are presented and discussed in terms of their importance and applicability in clinical practice. A total of 56 studies were included in this review. Overall, LC depth (LCD) and thickness (LCT) have been the most studied features, appearing in $75 \%$ and $45 \%$ of the included studies, respectively. These biomarkers were followed by the prelaminar tissue thickness (21\%), LC curvature index (5.4\%), LC global shape index (3.6\%), LC defects (3.6\%), and LC strains/deformations (1.8\%). Overall, the disease groups showed a thinner LC (smaller LCT) and a deeper ONH cup (larger LCD), with some exceptions. A large variability between approaches used to compute LC biomarkers has been observed, highlighting the importance of having automated and standardized methodologies in LC analysis. Moreover, further studies are needed to identify the pathologies where LC features have a diagnostic and/or prognostic value.
\end{abstract}

\section{Introduction}

The lamina cribrosa (LC) is a mesh-like structure localized in the posterior scleral canal of the optic nerve head $(\mathrm{ONH})$, allowing retinal ganglion cell (RGC) axons to pass through to the brain. It is a fenestrated complex that also accommodates vessels that nourish the retina. A large circumpapillary ring of collagen and elastin fibers, in the immediate peripapillary sclera, protects the LC against the mechanical strain, such as that induced by an imbalance between intraocular pressure (IOP) and intracranial pressure (ICP) $[1,2]$. Due to its anatomical location, between two differently pressurized compartments, there is a pressure gradient along the LC, denominated translaminar pressure difference (TLPD), which can be calculated as the difference between IOP and ICP in the subarachnoid space (SAS) [3, 4]. Despite 
being an extremely relevant structure to the eye's anatomy and function, little is known about the LC. LC morphology plays an important role in the development and progression of ophthalmic pathologies, notably on glaucomatous optic neuropathy, intraocular hypertension, and myopia [5-8]. The structural deformation and the correlated compression across the LC lead to blockade of axonal transport and eventually RGC death [9].

Recent advances in in vivo medical imaging techniques, such as optical coherence tomography (OCT), have allowed the visualization of deep connective tissues, including the LC, in greater detail (Figure 1) $[10,11]$.

Specific developments in OCT software, such as enhanced depth imaging (EDI), and light-attenuation correction software such as adaptive compensation (AC) significantly improved the visibility of the LC without compromising acquisition time. EDI-OCT was originally developed in order to improve the visualization of the choroid, although it has also been adopted to improve crosssectional images of the LC. AC is a postprocessing technique developed to remove blood vessel shadows and enhance tissue contrast in order to facilitate posterior LC surface detection [12, 13]. In addition to these software developments, several studies have shown that swept-source OCT (SS-OCT) further improves the visualization of the LC $[14,15]$.

While an increasing number of works have studied the relevance of the LC (and its changes) in glaucoma [16], data on other diseases are still scarce. Hence, this review intends to provide a broader vision and a better comprehension of the measurable laminar structural features that have been identified as relevant for nonglaucomatous pathologies in the published literature.

\section{Methods}

2.1. Study Selection. A literature search was conducted in the MEDLINE (PubMed) bibliographic database on 15th May 2020. The search query was (optical coherence tomography NOT angiography) AND (lamina OR cribrosa). Only articles published in English were considered, and no publication date restriction was added. The exclusion criteria were (i) only included glaucomatous eyes in the experimental group; (ii) not conducted in humans; (iii) review articles or case reports; (iv) exclusive focus on imaging techniques and not presenting clinical data; (v) no evaluation of the lamina cribrosa; and (vi) no mention to LC structural parameters and how they were measured/extracted. This led to a total of 408 references, which were narrowed down to 56 after title/ abstract screening, followed by a full-text screening (see Figure 2). The 56 included studies provided quantitative values for each of the analyzed features and described how the quantification was performed.

2.2. Data Collection. In this review, our main aim was to identify potential biomarkers in the morphology of the LC that were associated with, and indicative of, certain pathologies. Therefore, we have opted to only report those that performed a statistical comparison between an experimental and a control group. The extracted data for each paper consist of the LC structural parameters, their mean and standard deviation (SD), and the $p$ values of the statistical analysis performed between experimental and control groups. Moreover, the image processing methodology applied to compute each feature was taken into account for posterior comparisons.

For all the included articles, the following characteristics are obtained and presented in Table 1: sample size, including the number of patients and eyes per group; age and statistical comparison ( $p$ value) between control and experimental groups; OCT device model, manufacturer, and light-source wavelength; cutoff value for the signal strength index (SSI) or similar qualitative image criteria used to exclude patients/ eyes; and field of view of the OCT image [17]. Moreover, the procedures followed to measure the LC features, and their respective values, were also collected and compiled.

Data collection comprehended all structural components related to the LC and the surrounding $\mathrm{ONH}$ region that were included on the OCT B-scan images. Several locations, planes (superior, middle, and inferior), and sectors were considered for the measurements. The sectors were defined according to the Garway-Heath map [71]. The approach of data extraction by one investigator (ASP) with further verification by a senior author (JBB) has been used, as this has been demonstrated to be as accurate as double independent data extraction [72].

2.3. Data Analysis. The obtained data were used to calculate the frequency of each LC structural feature in the published literature and to determine the mean values of the most frequently reported features. Statistical relevance, given by the $p$ value, was also taken as a complement for study results and to comprehend the relation and differences found between study groups. To average the data for each LC parameter, pooled mean and pooled SD were determined according to equations (1) and (2), respectively, where $N$ represents the number of eyes included in the study, $M$ the mean value, $\mathrm{SD}$ the standard deviation, and $n$ the number of studies analyzed:

$$
\begin{aligned}
\text { pooled mean } & =\frac{\sum_{i=1}^{n} N_{i} \times M_{i}}{\sum_{i=1}^{n} N_{i}}, \\
\text { pooled SD } & =\sqrt{\frac{\sum_{i=1}^{n}\left(N_{i}-1\right) \times \mathrm{SD}_{i}^{2}}{\sum_{i=1}^{n}\left(N_{i}-1\right)}} .
\end{aligned}
$$

\section{Results}

All structural LC features were analyzed, and the studies were organized in three groups: healthy group $(n=19)$, nonophthalmic disease group $(n=6)$, and ophthalmic (nonglaucomatous) disease group $(n=31)$. Overall, LC depth (LCD) and LC thickness (LCT) have been the most studied features, appearing in $75 \%$ and $44.6 \%$ from the total articles. Other features, such as prelaminar tissue thickness 


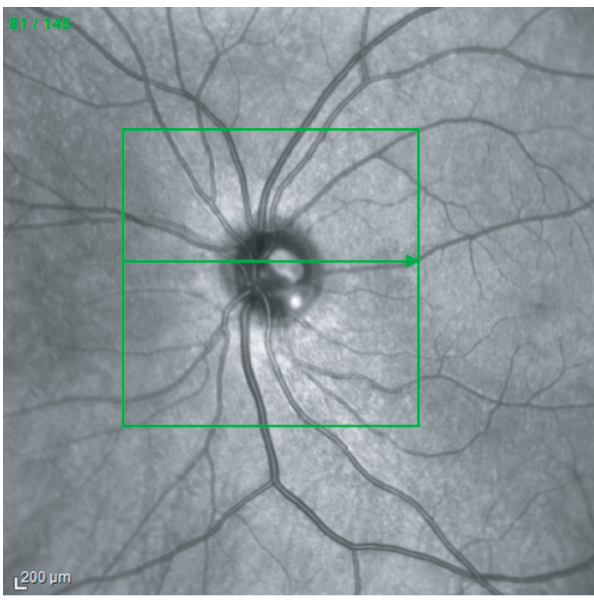

(a)

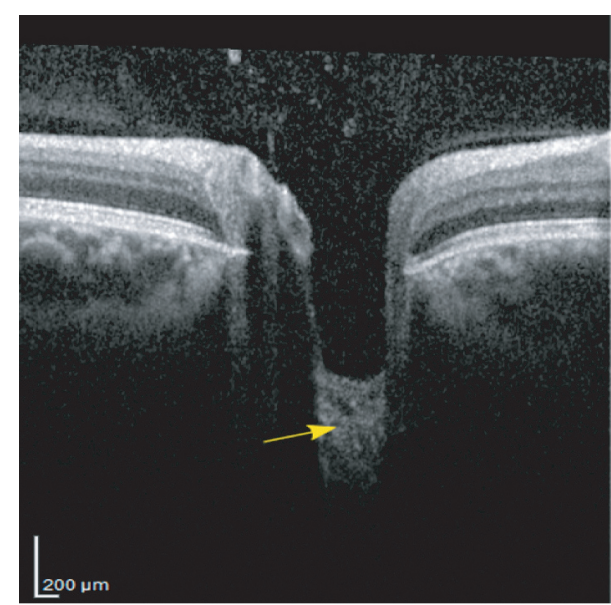

(b)

FIGURE 1: Retinal fundus photograph (a) and EDI-OCT B-scan at the optic nerve head (b). The green line denotes the location of the B-scan in the fundus image. The yellow arrow points to the lamina cribrosa region.

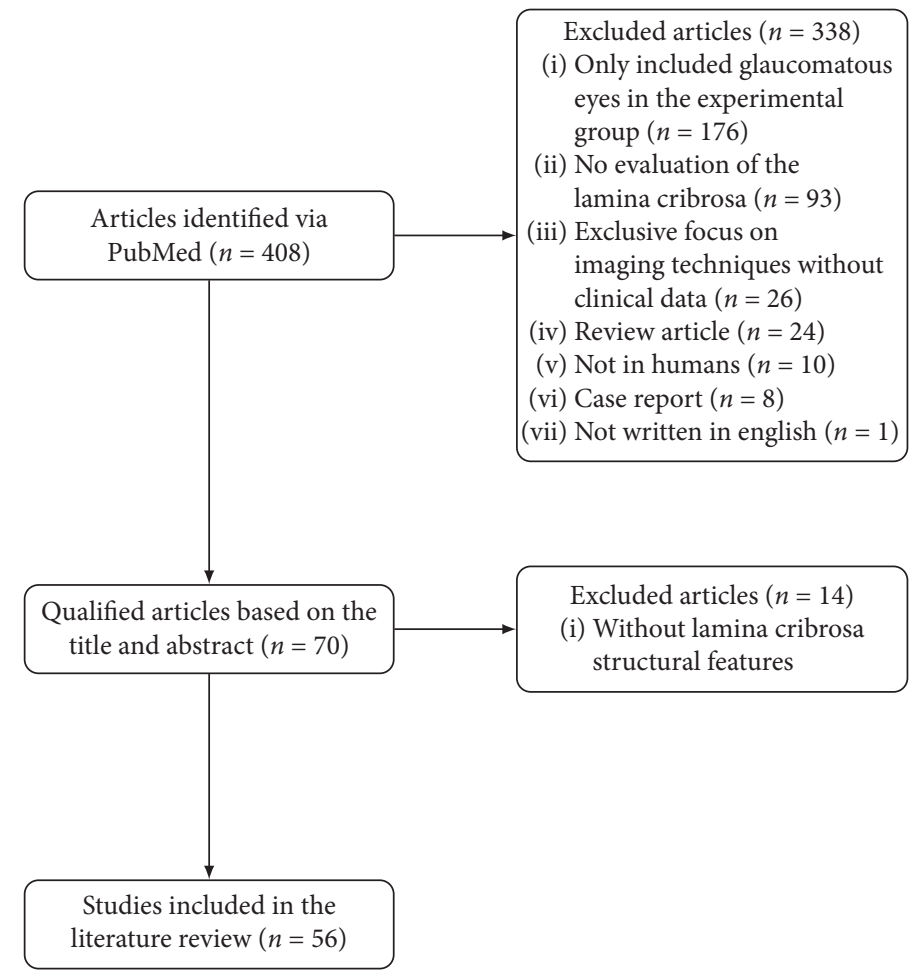

FIgURE 2: Flowchart of the collected data.

(PTT) (21.4\%) studied in ophthalmic and nonophthalmic diseases, and lamina cribrosa curvature index (LCCI) (5.4\%), LC global shape index (3.6\%), LC defects (3.6\%), slope of the LC (3.6\%), distance between the inner surface of the LC (3.6\%), SAS (3.6\%), and LC strains/deformations (1.8\%) studied in ophthalmic diseases only, are also referenced but in fewer studies.

One bar chart, summarizing the most studied features in the ophthalmic and nonophthalmic disease groups, is presented in Figure 3. Hence, in the following sections, a detailed analysis on how these two biomarkers have been measured is provided. Moreover, a detailed explanation on how LCD and LCT measurements were carried out in each study is presented in Table 2 . The normative values for the groups are also presented and discussed. In cases where more than one measurement was performed for the same feature (e.g., in different planes (superior, middle, or inferior), different scan directions (vertical and horizontal), or 2 eyes (left and right)), the pooled mean and SD were determined according to equations (1) and (2).

Only diseases for which at least 20 eyes were included in the studied group (cumulatively over all the evaluated 


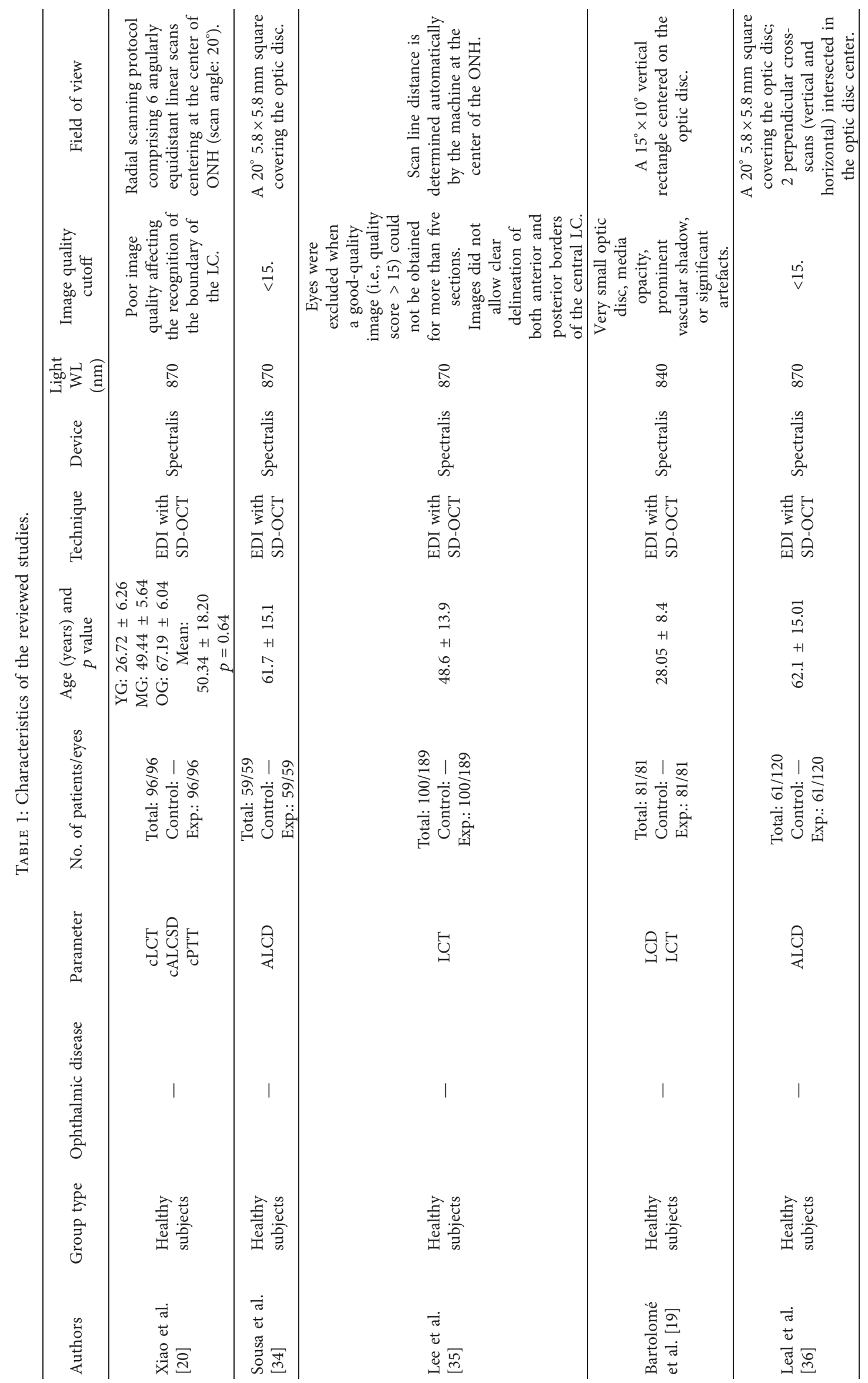




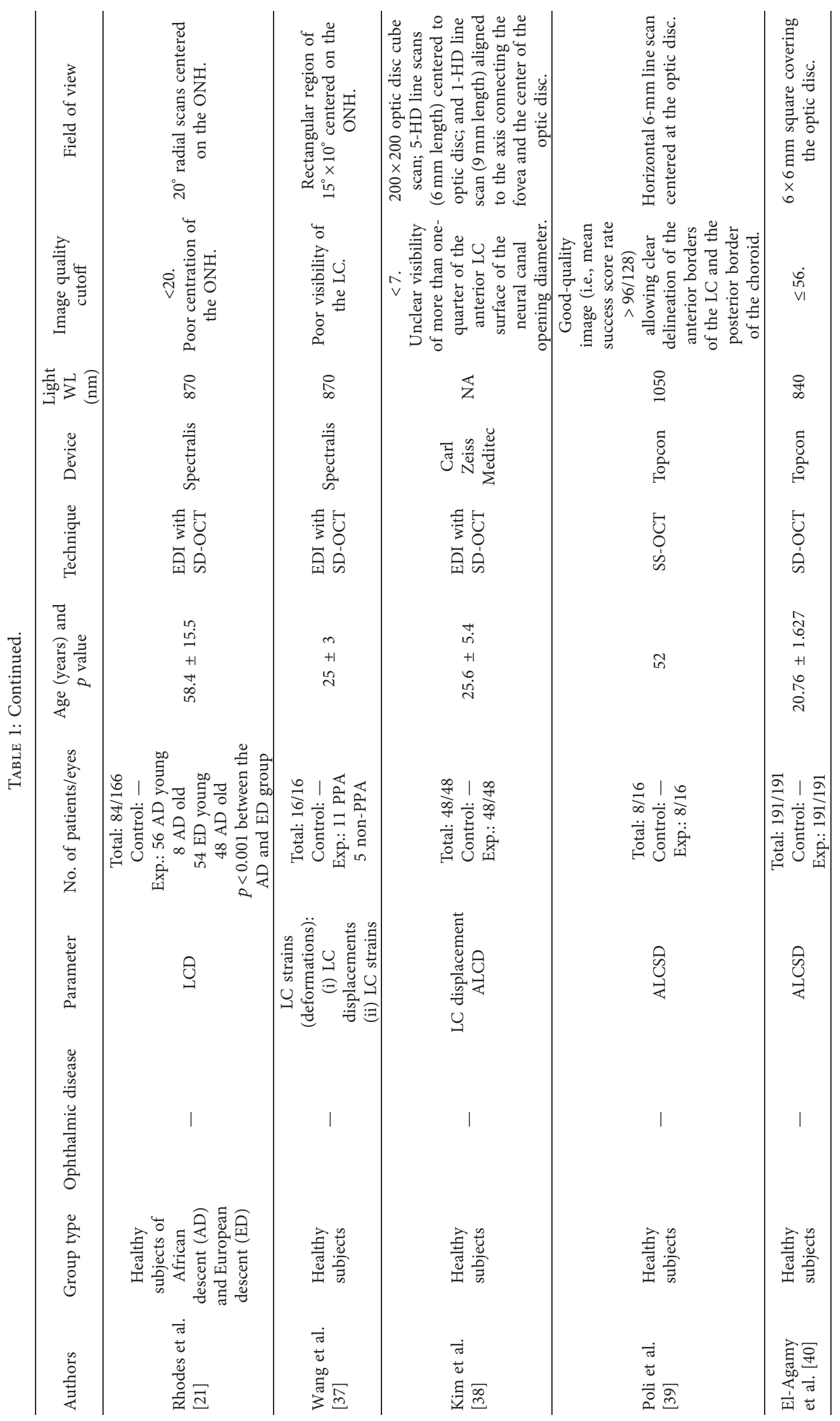




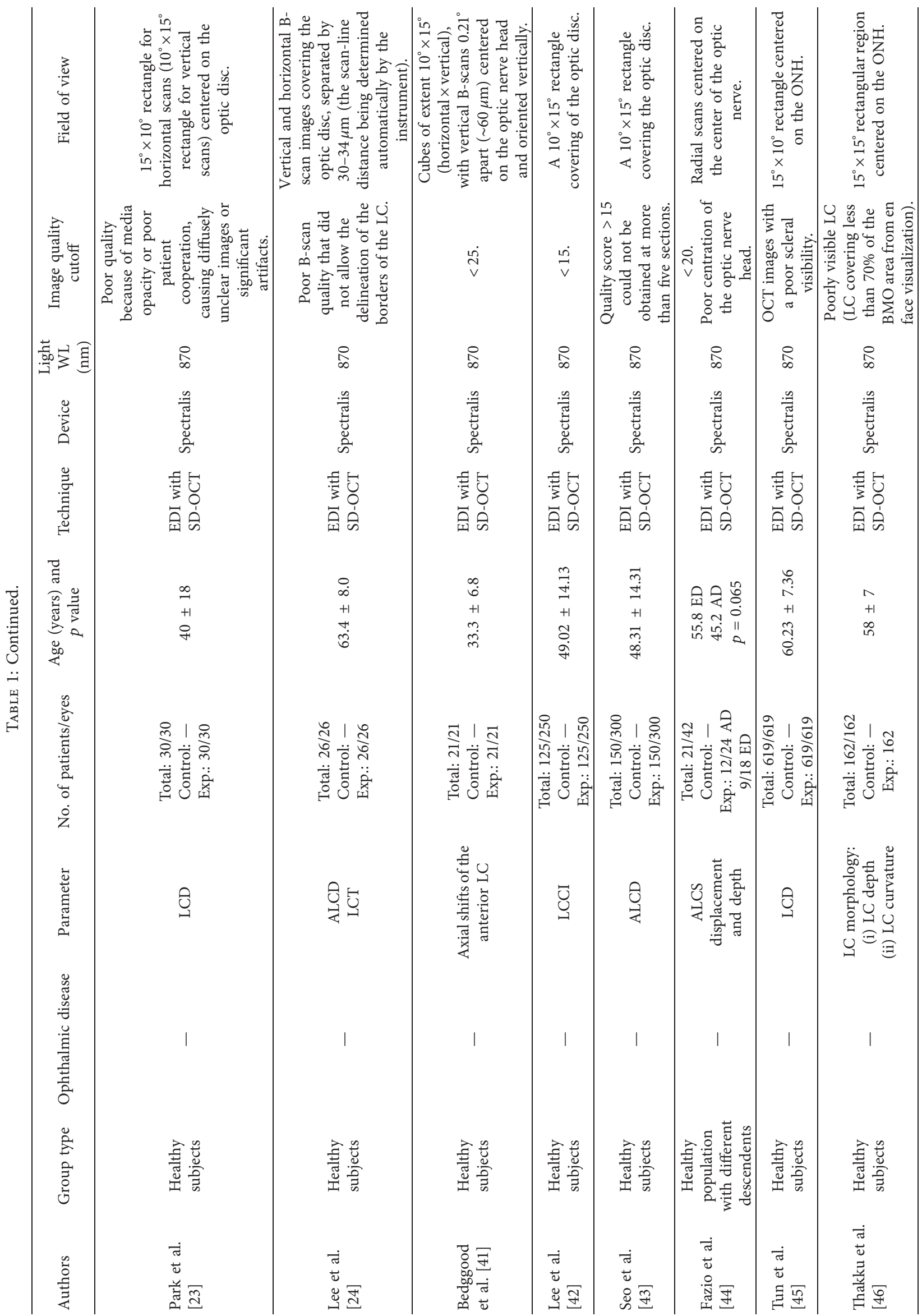




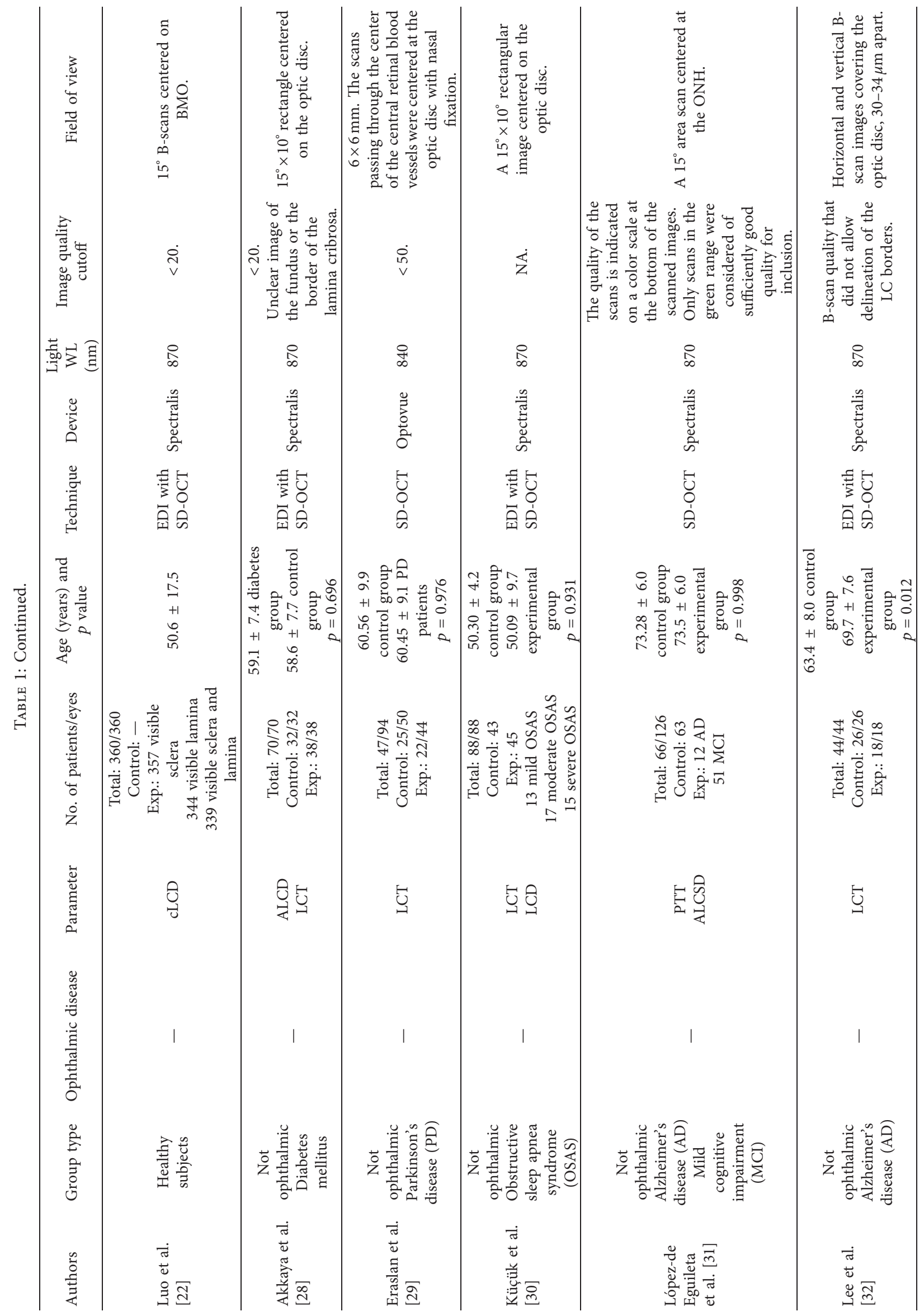




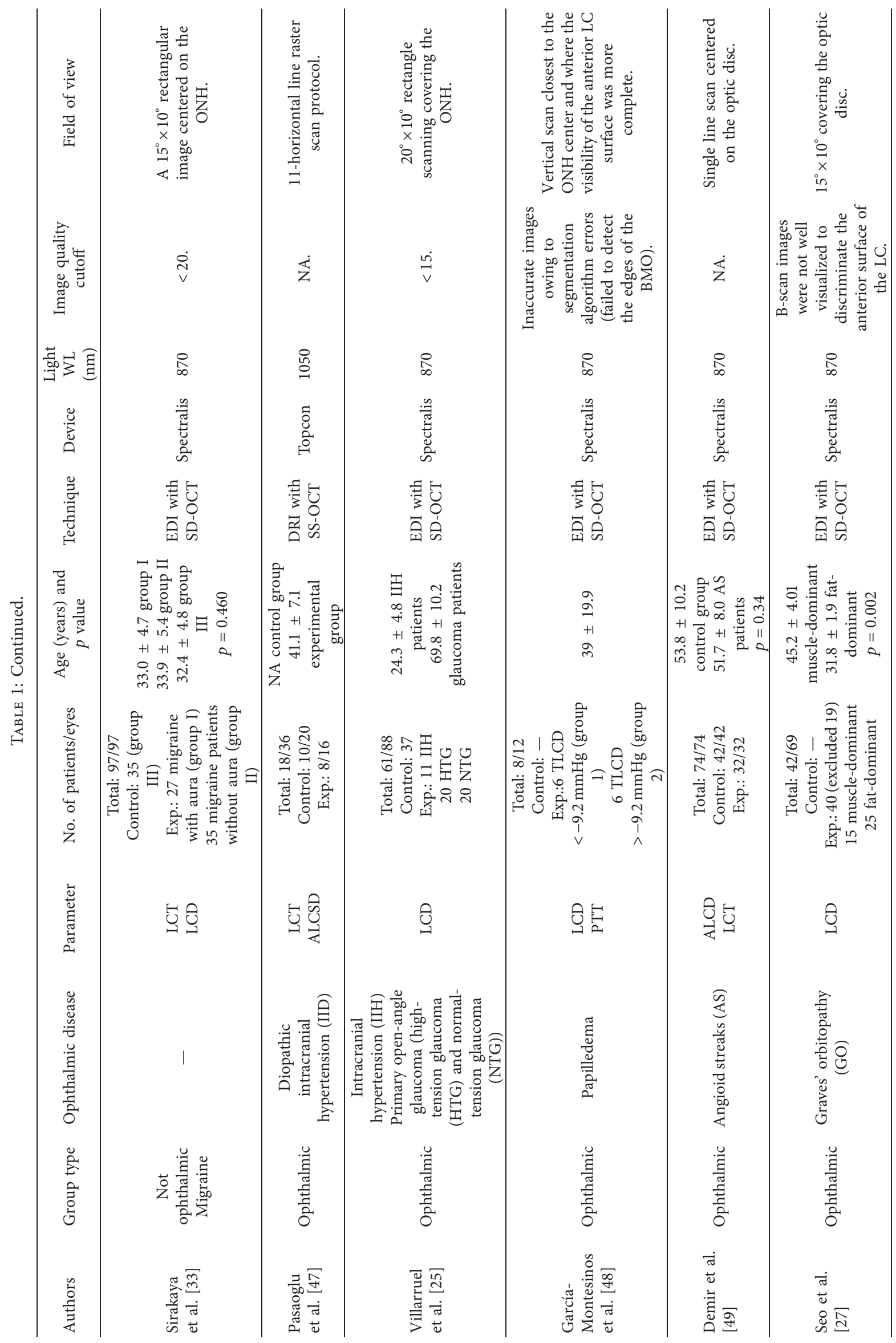




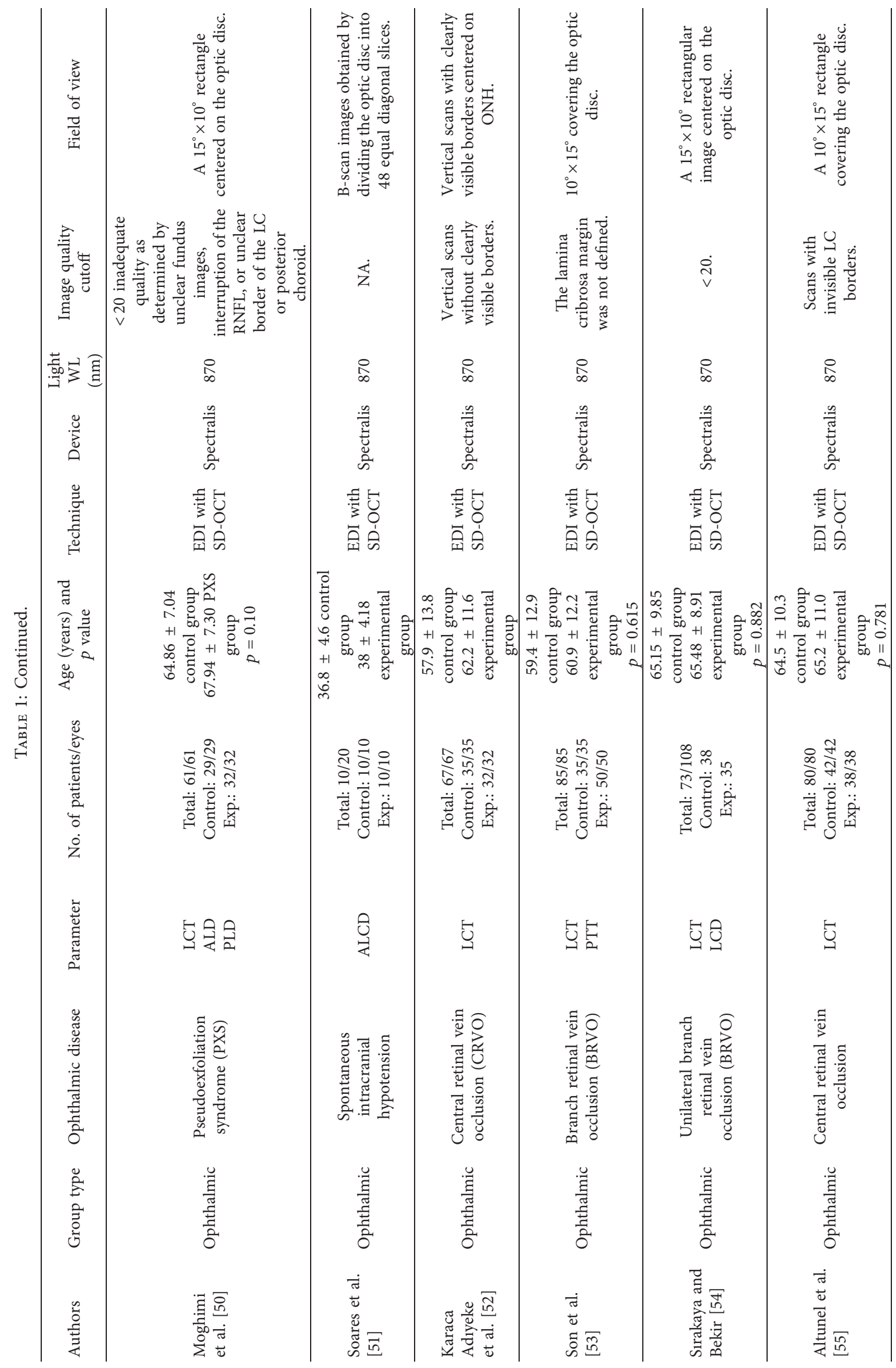




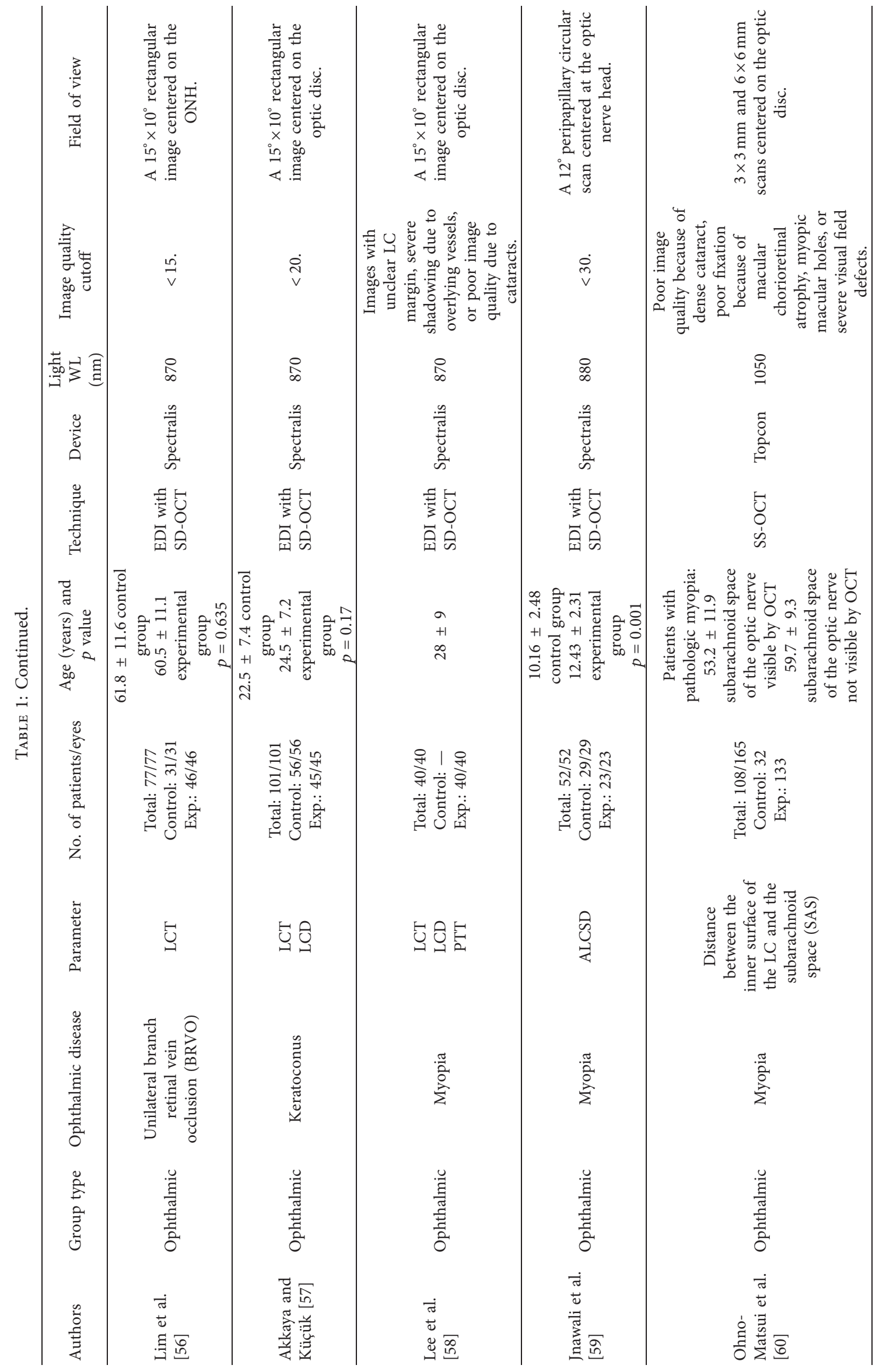




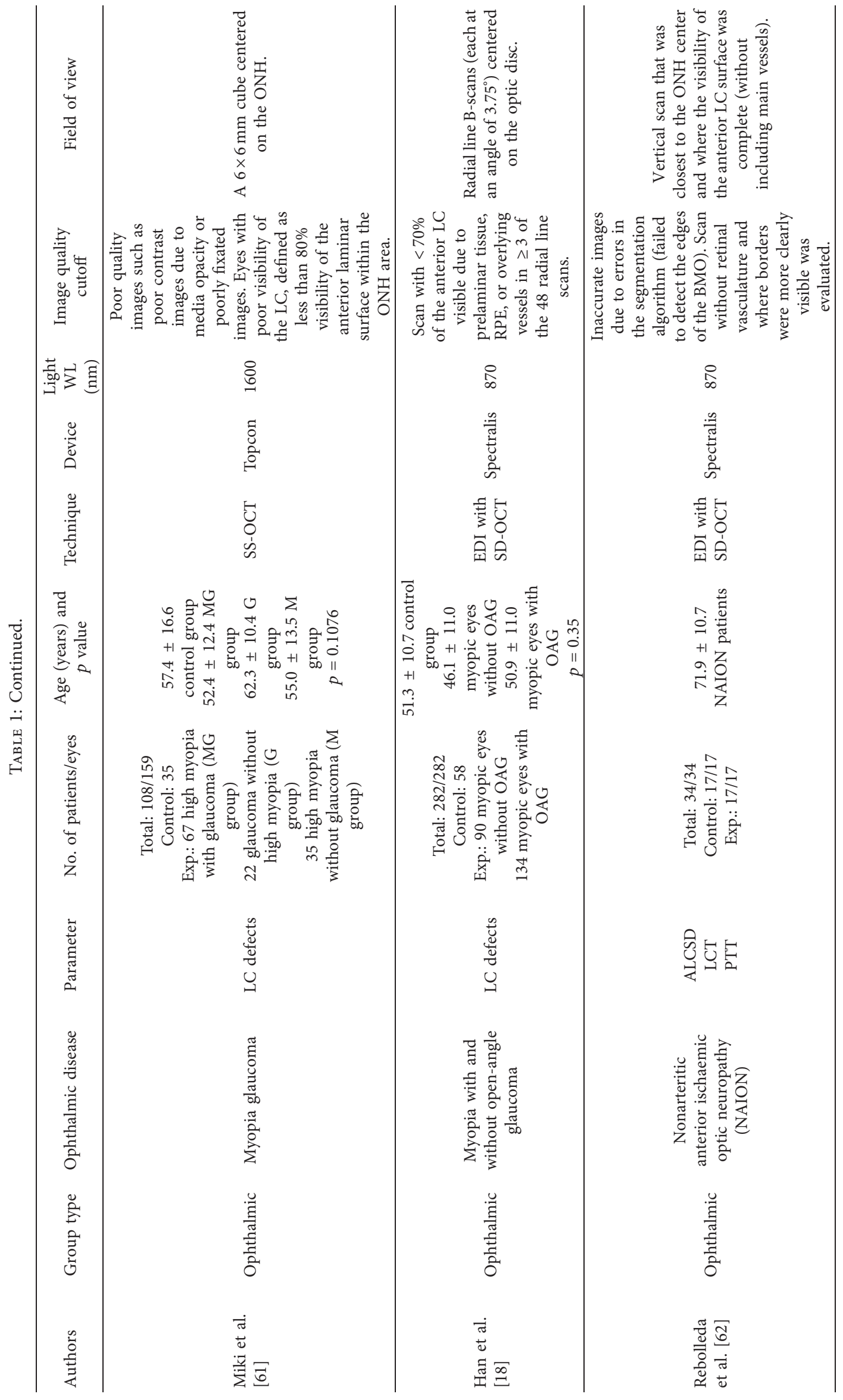




\begin{tabular}{|c|c|c|c|c|c|}
\hline 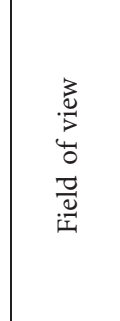 & 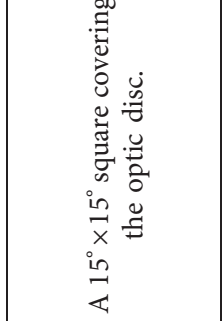 & 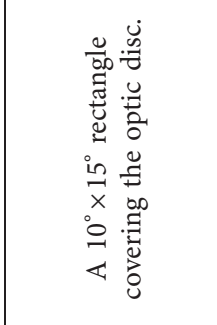 & 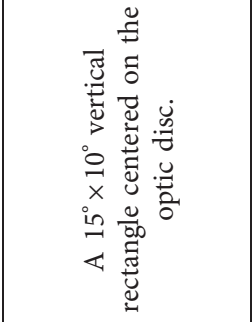 & 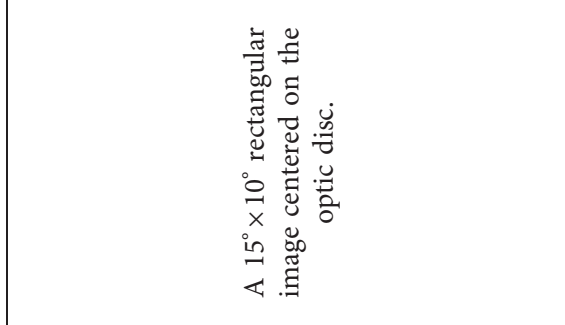 & 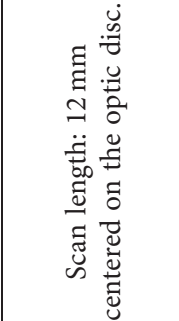 \\
\hline 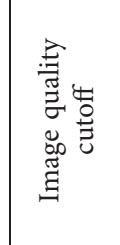 & 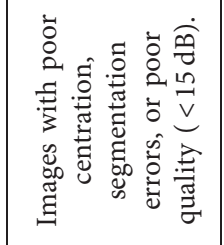 & 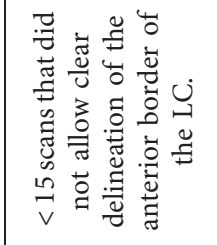 & 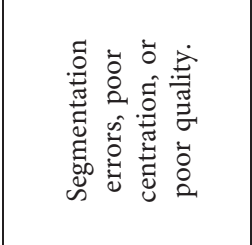 & 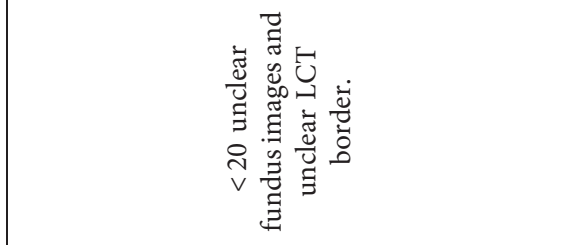 & 崩 \\
\hline 营字毛 & $\underset{\infty}{\stackrel{R}{\infty}}$ & 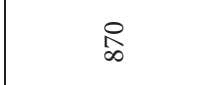 & 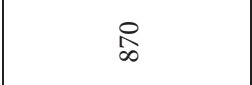 & $\underset{\infty}{\stackrel{0}{\infty}}$ & 옹 \\
\hline 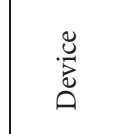 & 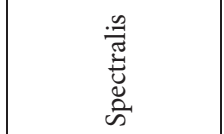 & 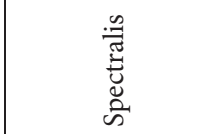 & 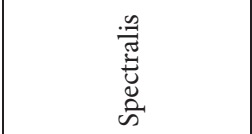 & 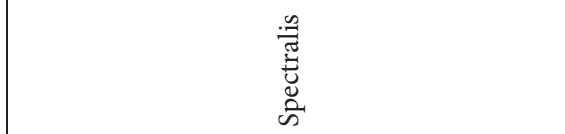 & 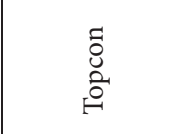 \\
\hline 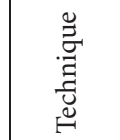 & 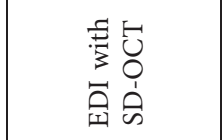 & 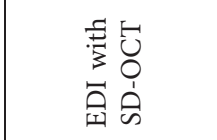 & 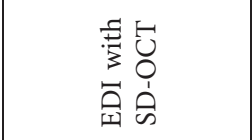 & 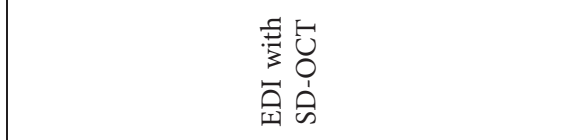 & $\begin{array}{l}y_{0} \\
0 \\
\dot{s}\end{array}$ \\
\hline 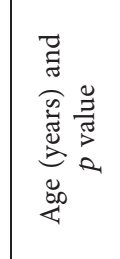 & 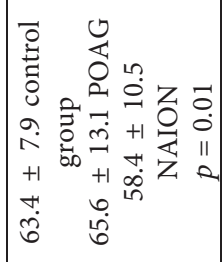 & 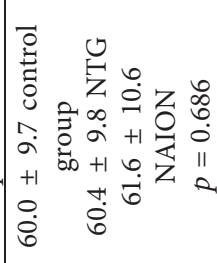 & 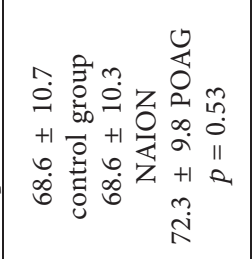 & 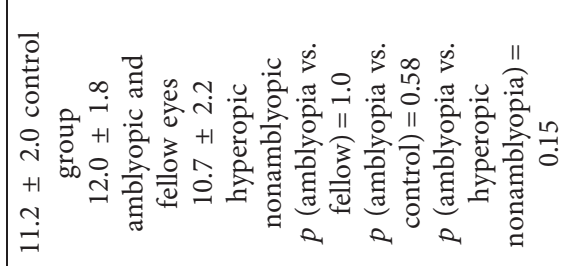 & 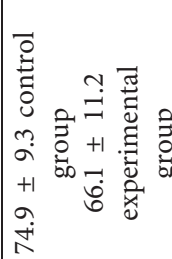 \\
\hline 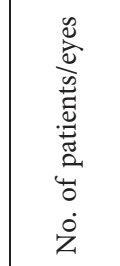 & 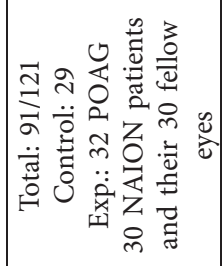 & 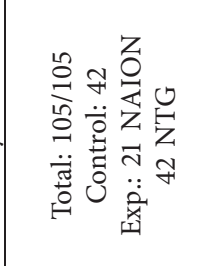 & 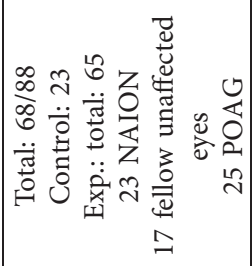 & 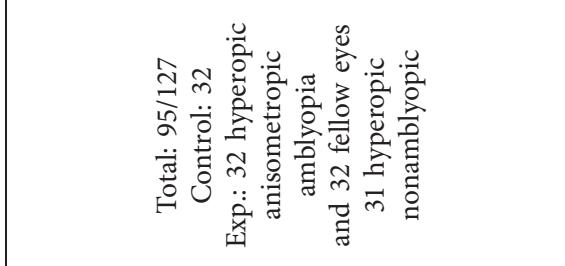 & 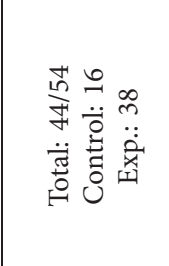 \\
\hline 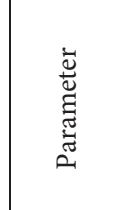 & 岂 & 睍 & 䙷㤩 & 舅兽 & 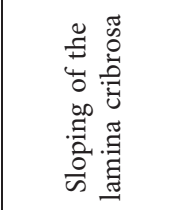 \\
\hline 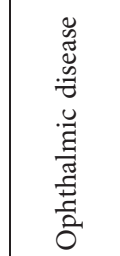 & 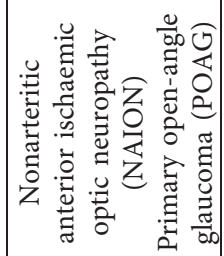 & 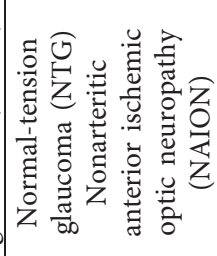 & 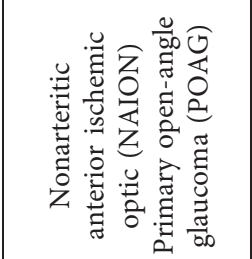 & 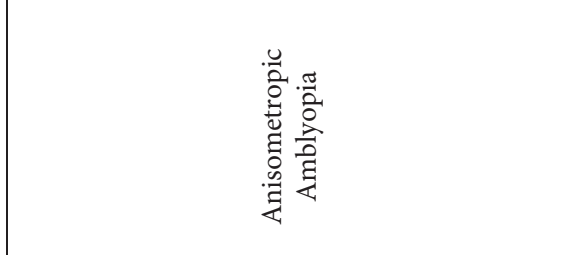 & 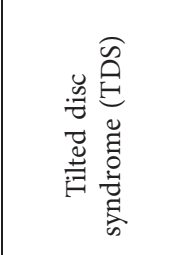 \\
\hline 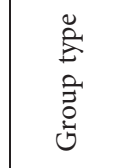 & 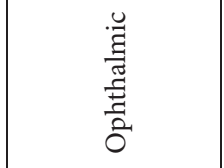 & 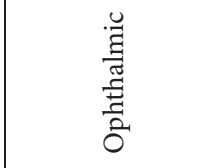 & 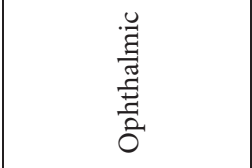 & 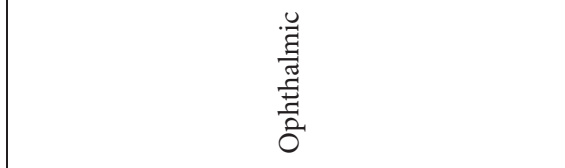 & 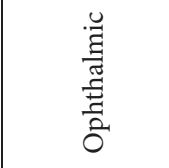 \\
\hline 妾 & 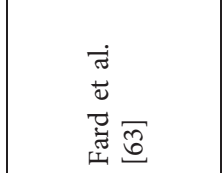 & 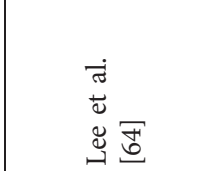 & 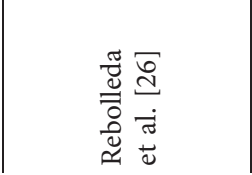 & 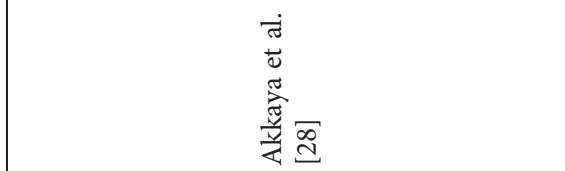 & 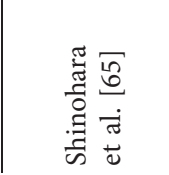 \\
\hline
\end{tabular}




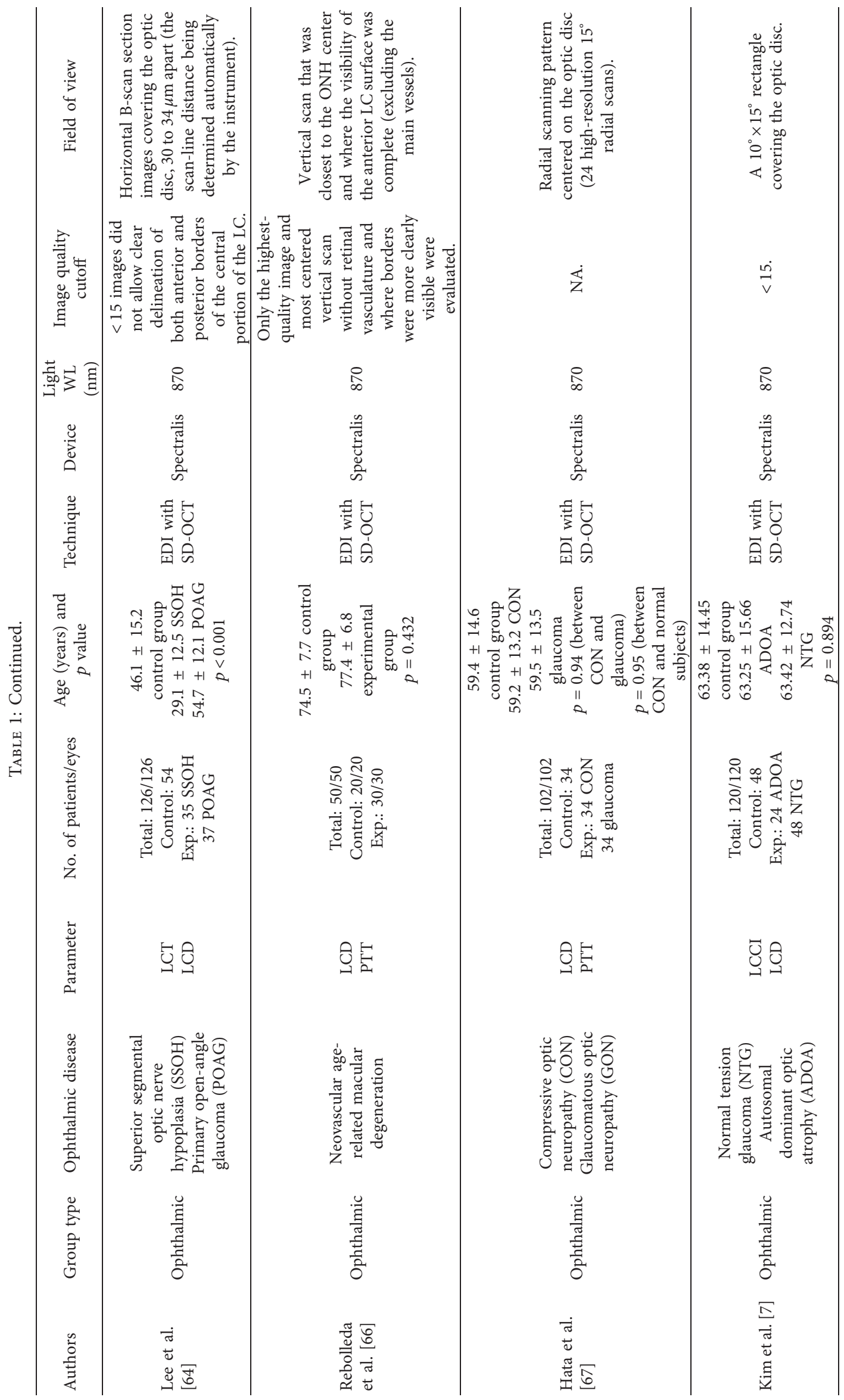




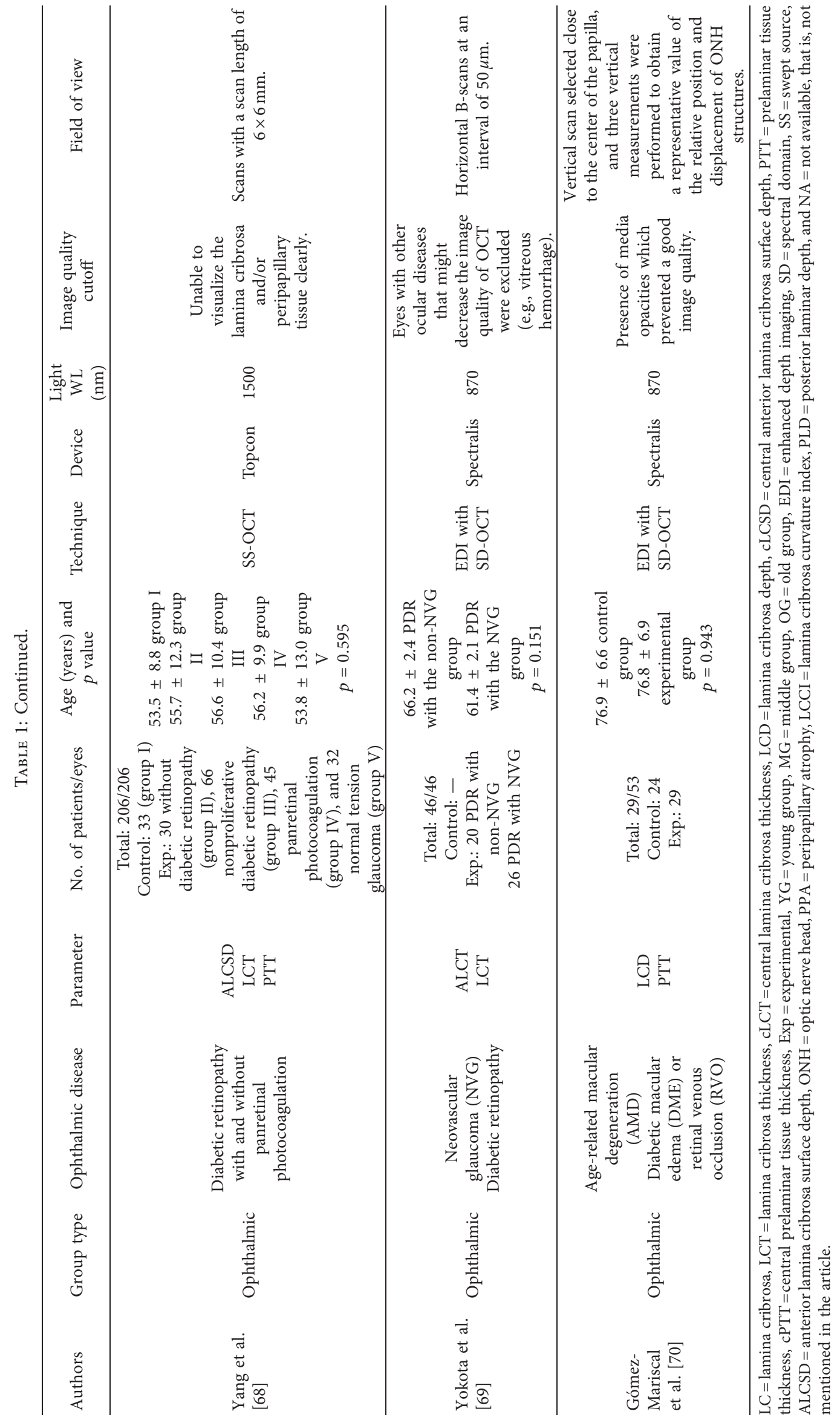




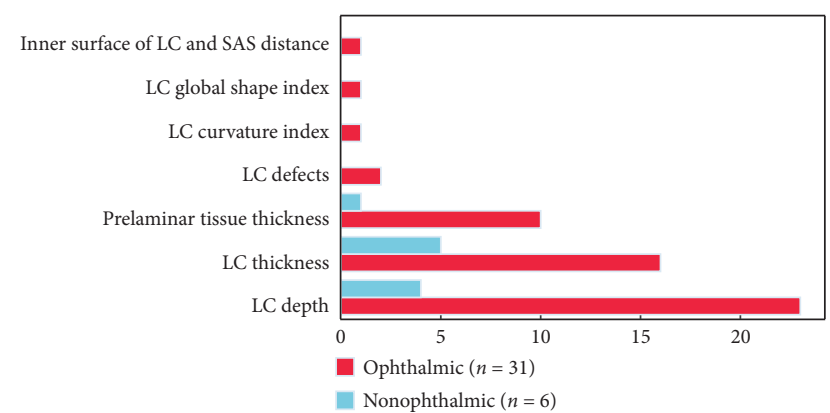

Figure 3: Number of occurrences of each lamina cribrosa structural feature published in the literature. The value $n$ corresponds to the total number of studies for each group.

papers) were considered for the average calculations (equations (1) and (2)).

3.1. LCT Measurements. LCT has been defined in the literature as the distance between the anterior and posterior borders of the highly reflective region visible below the optic disc cup in B-scan cross sections of the ONH (see the red arrow in Figure 4) [18]. However, a discrepancy between the LCT measurements, namely, between the locations used to calculate the LCT average, has been observed and reported in Table 2. For example, Lee et al. [74] considered three locations in each eye (midhorizontal, superior, and inferior midperipheral), with a separation of $100 \mu \mathrm{m}$ between the points. Bartolomé et al. [19] determined the points as close as possible to the vertical center of the $\mathrm{ONH}$, which was identified as the point where the trunk of central retinal vessels extends from the $\mathrm{ONH}$, as reported by Park et al. [12]. Other authors, such as Xiao et al. [20], considered LCT as the average of the central and paracentral points $(150 \mu \mathrm{m}$ from the center point in the horizontal and vertical directions).

3.2. LCD Measurements. In the literature, LCD (also named as anterior lamina cribrosa depth in several studies) is defined as the perpendicular distance from the BMO plane to the maximum depth point of the anterior LC surface. All articles included in this review provide the measurements relative to Bruch's membrane opening (BMO). Only the measurements relative to $\mathrm{BMO}$ were considered for the calculations since all articles provide the measurement relative to this plane. Two studies, Rhodes et al. [21] and Luo et al. [22], also considered the scleral plane and the ASCO as the reference for depth measurements. These differences have been shown to lead to measurement bias, as reported by Luo et al. [22], who obtained $402 \pm 91 \mu \mathrm{m}, 309 \pm 88 \mu \mathrm{m}$, and $332 \pm 96 \mu \mathrm{m}$ for BMO, ASCO, and scleral reference planes, respectively. The number of selected points and Bscan planes to average the measure also influence the precision of the results. Other authors, such as Park et al. [23], obtained measurements as the average from 11 equidistant planes that divided the optic disc diameter into 12 equal parts vertically in each eye. A line was drawn from each of the two LC insertion points perpendicularly to the line connecting the two Bruch's membrane edges (see line D in Figure 4). The area surrounded by these two lines was measured (see area S in Figure 4). The mean LCD was approximated by dividing $S$ by the length of $\mathrm{D}$ for each of the 11 horizontal OCT scans. Finally, Lee et al. [24] defined LCD as the mean of 3 values obtained from the 3 upper B-scans (1st to the 3 rd scan), the 3 central B-scans (5th to the 7 th scan), and at the 3 lower B-scans (from the 9th to the 11th scan) passing through the ONH. Commonly, temporal adjacent points were selected because the maximally depressed point was often close to the central vessel trunk, and its shadow obscured the LC [25].

3.3. Features' Applicability and Measurements. This section details the mean values for the two dominating LC structural features (LCT and LCD) in the three groups (healthy controls, ophthalmic, and nonophthalmic diseases). The values were calculated based on the articles presented in Table 2 for each group and disease, and the mean and SD values for each group are summarized in Figure 5.

3.3.1. Healthy Group Measurements. Analysis of healthy subjects is very important to establish normative values for the healthy population, and hence facilitate the diagnosis and follow-up of the pathology. The studies that included only healthy subjects, as well as those comparing patients to a healthy control group, were selected, and the LCT and LCD average values were determined. The observed averages were $261 \pm 39 \mu \mathrm{m}$ (range: $211-323 \mu \mathrm{m}$ ) and $386 \pm 91 \mu \mathrm{m}$ (range: 293-441 $\mu \mathrm{m}$ ) for the LCT and LCD, respectively. Figures 5(a) and 5(b) show a comparison between the three groups for both features. These parameters seem to be influenced by several factors, such as age and racial ancestry. Rhodes et al. [21] conducted a study in healthy eyes and concluded that the LC was significantly anteriorly displaced with increasing age in those with European ancestry in contrast to those with African ancestry.

3.3.2. Ophthalmic Disease Group Measurements. The ophthalmic disease group represented the largest group $(n=31)$ and included a large number of conditions, the most common being myopia, retinal vein occlusion (RVO), nonarteritic anterior ischaemic optic neuropathy (NAION), pseudoexfoliation syndrome (PXS), superior segmental optic nerve hypoplasia (SSOH), compressive optic neuropathy $(\mathrm{CON})$, age-related macular degeneration (AMD), autosomal dominant optic atrophy (ADOA), and diabetic macular edema (DME). For ophthalmic patients, mean LCT and LCD were $211 \pm 33 \mu \mathrm{m}$ and $403 \pm 90 \mu \mathrm{m}$, respectively (Figure 5). The graphics in Figure 6 presents the mean values for different ophthalmic diseases in comparison with the healthy population (horizontal dashed green line). Regarding the LCT, its mean was lower for every pathology in this group except for SSOH (Figure 6(a)). Overall, the studies that reported LCD in nonglaucomatous ophthalmic diseases showed a slightly higher mean LCD compared with healthy controls (Figure 5(b)). Nonetheless, this trend is not 


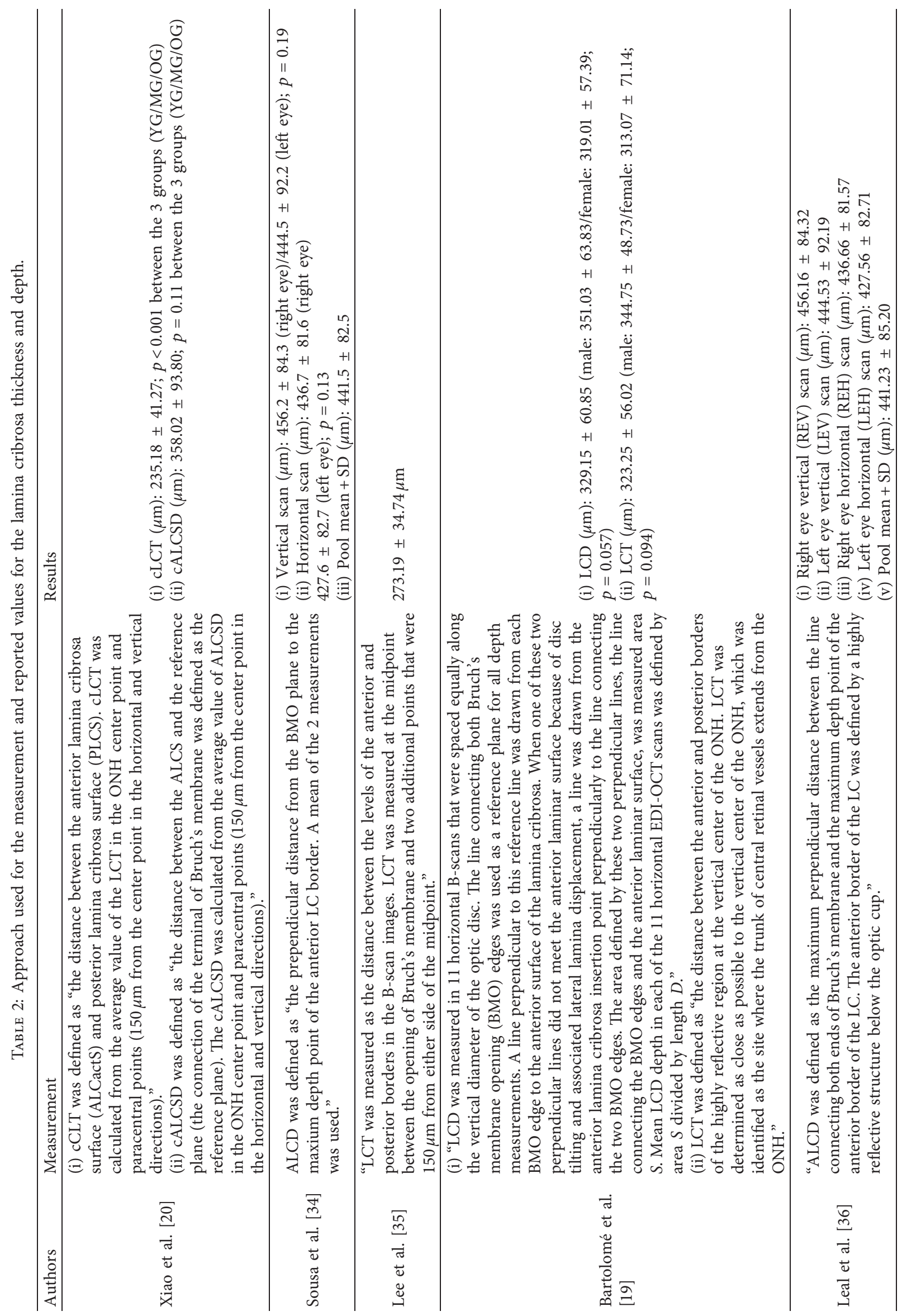




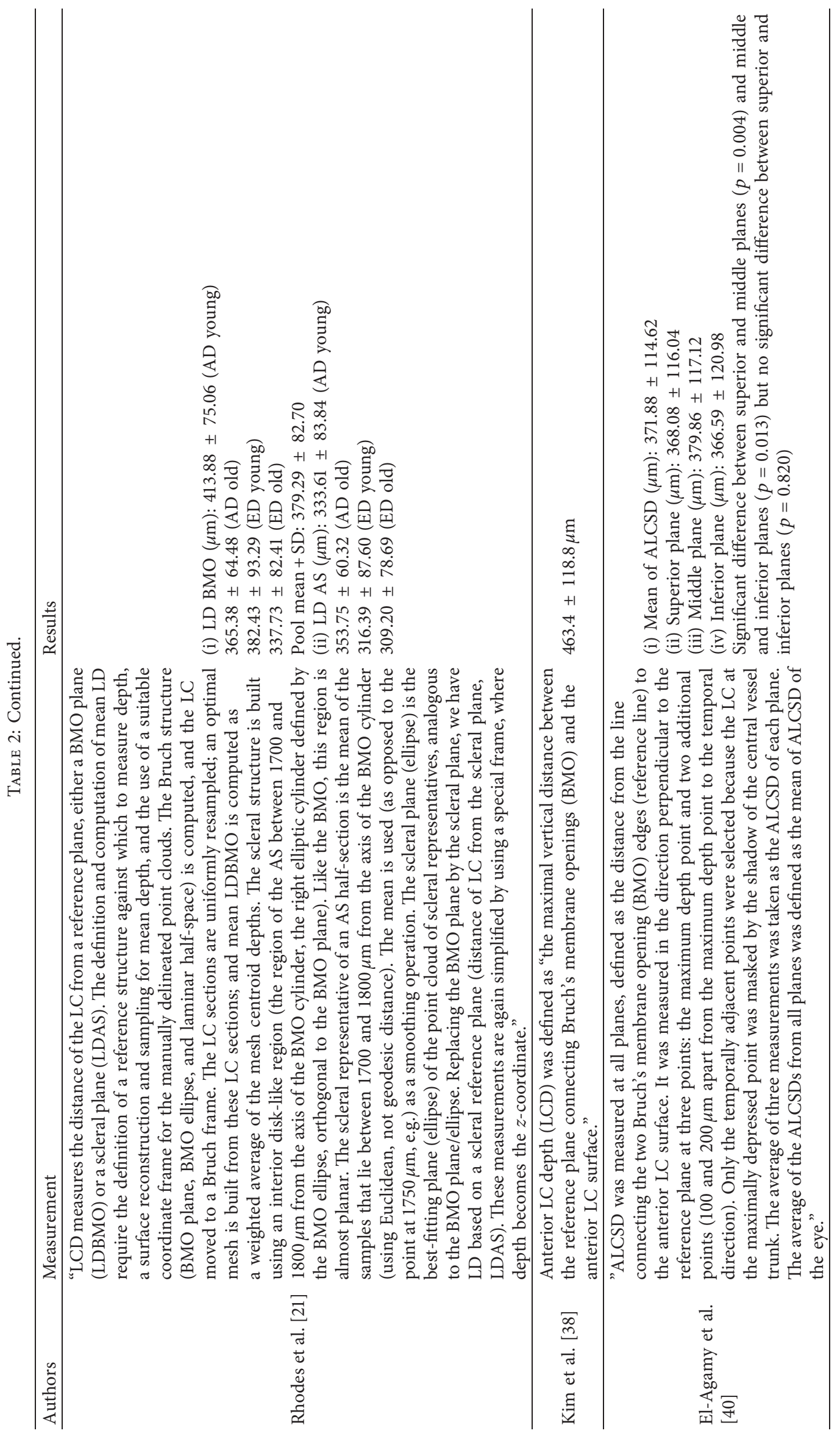




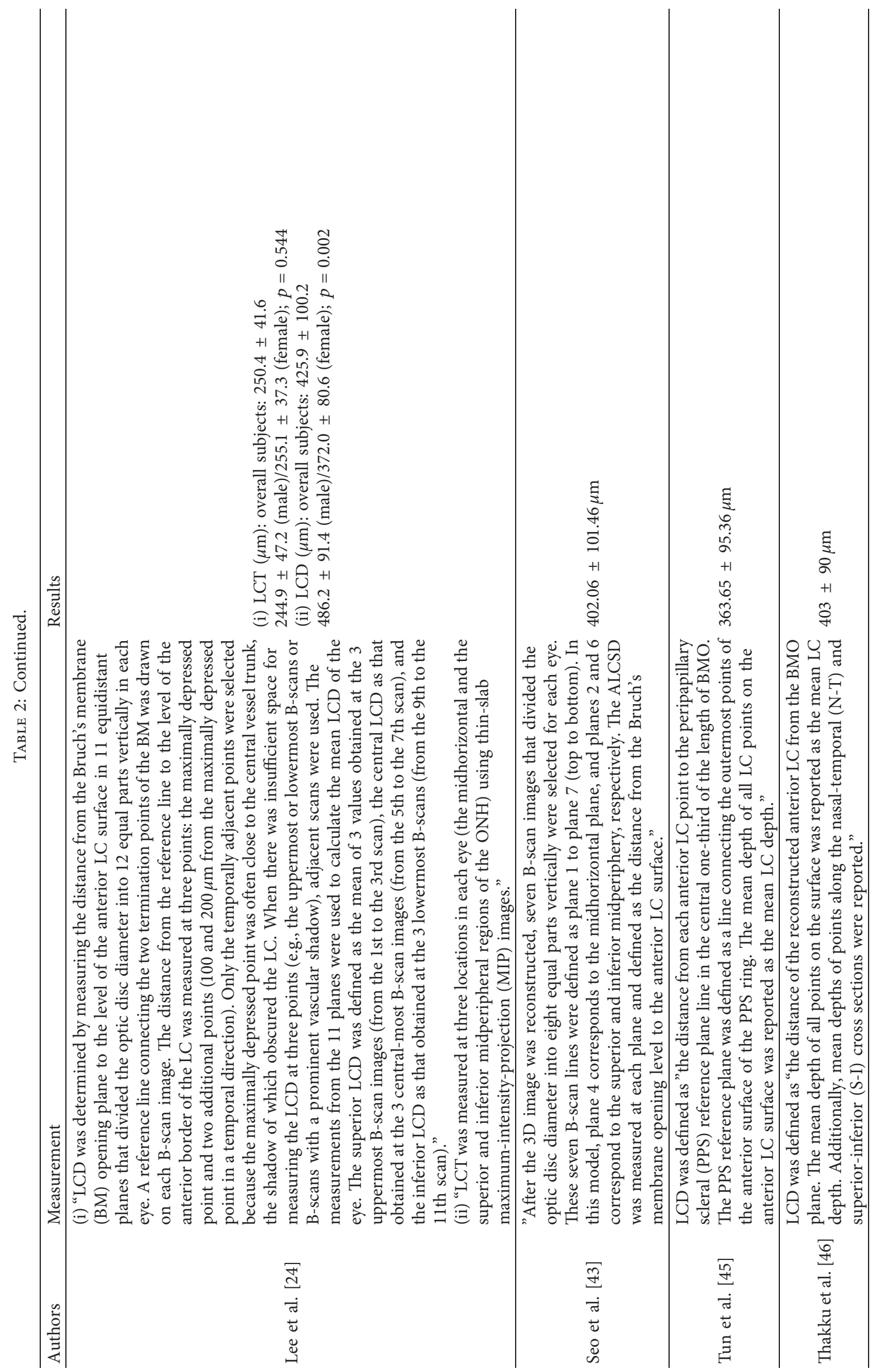




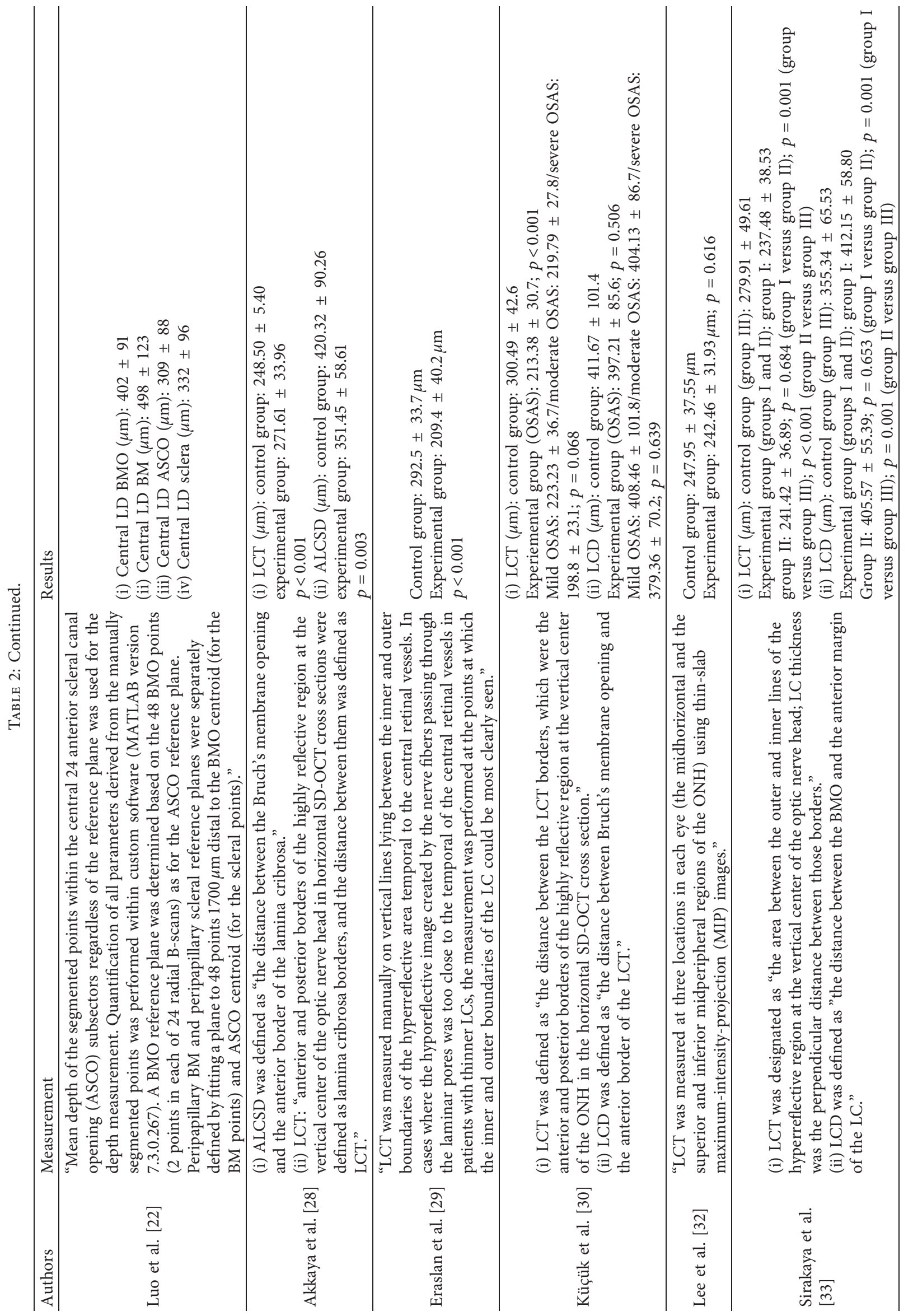




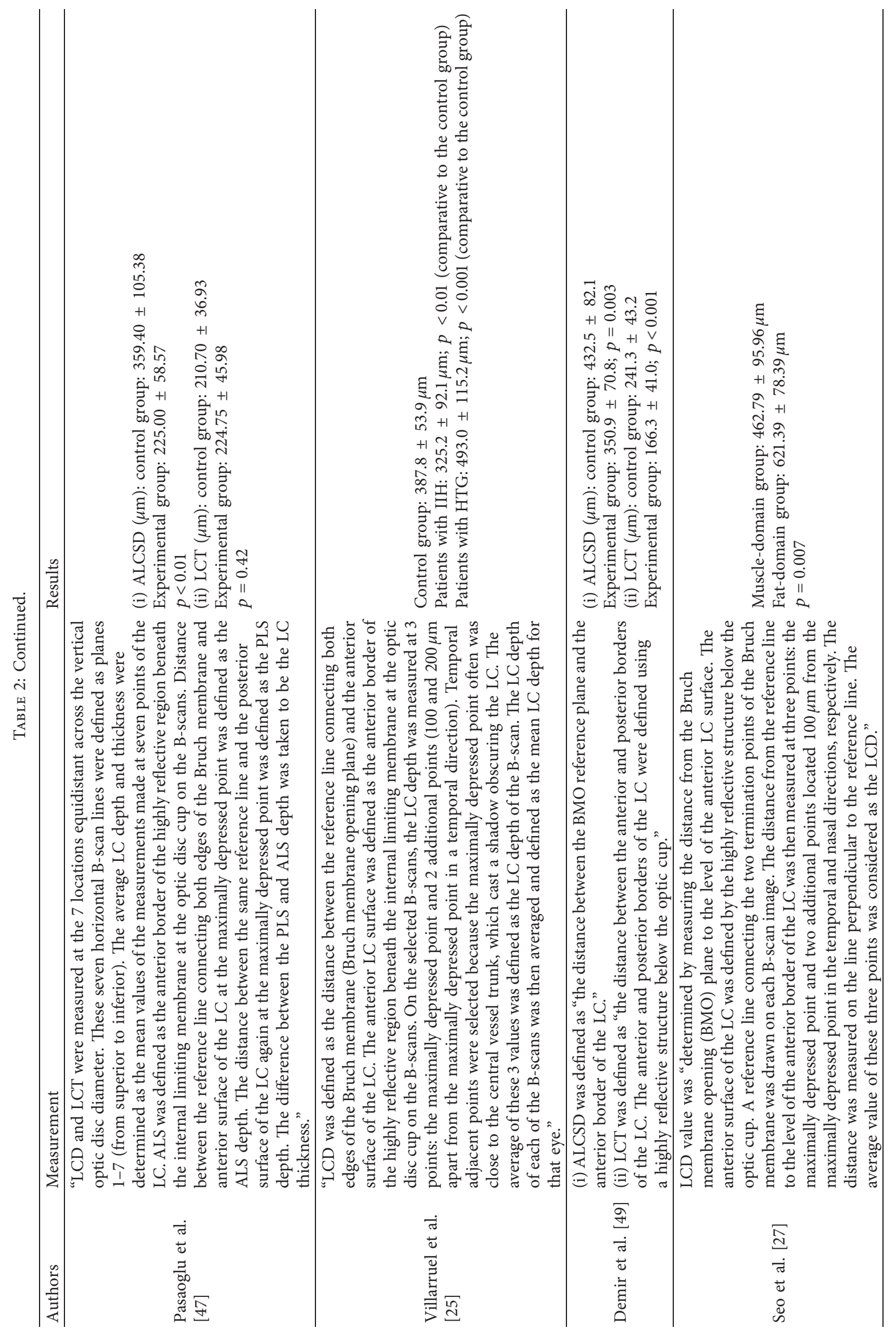




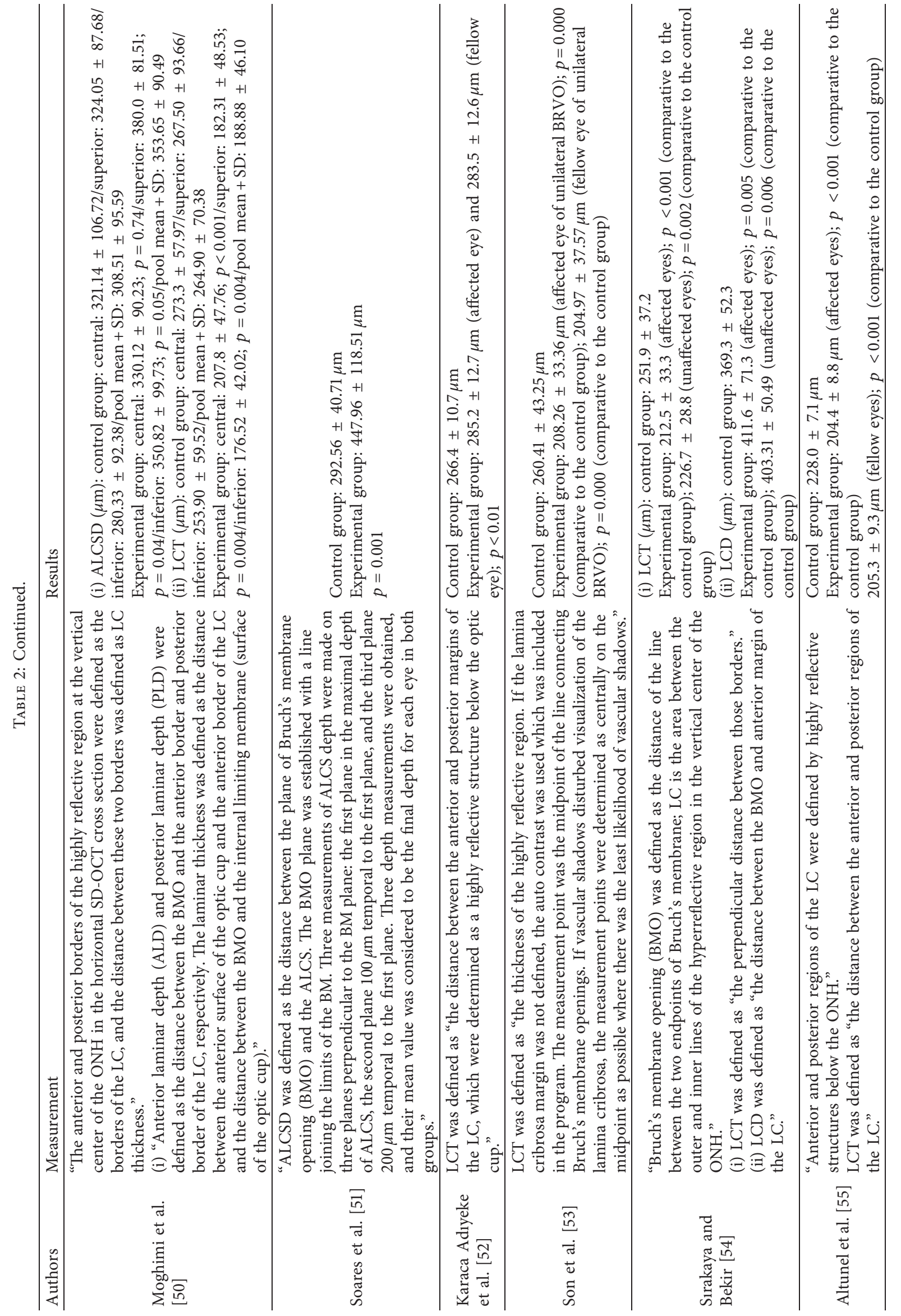




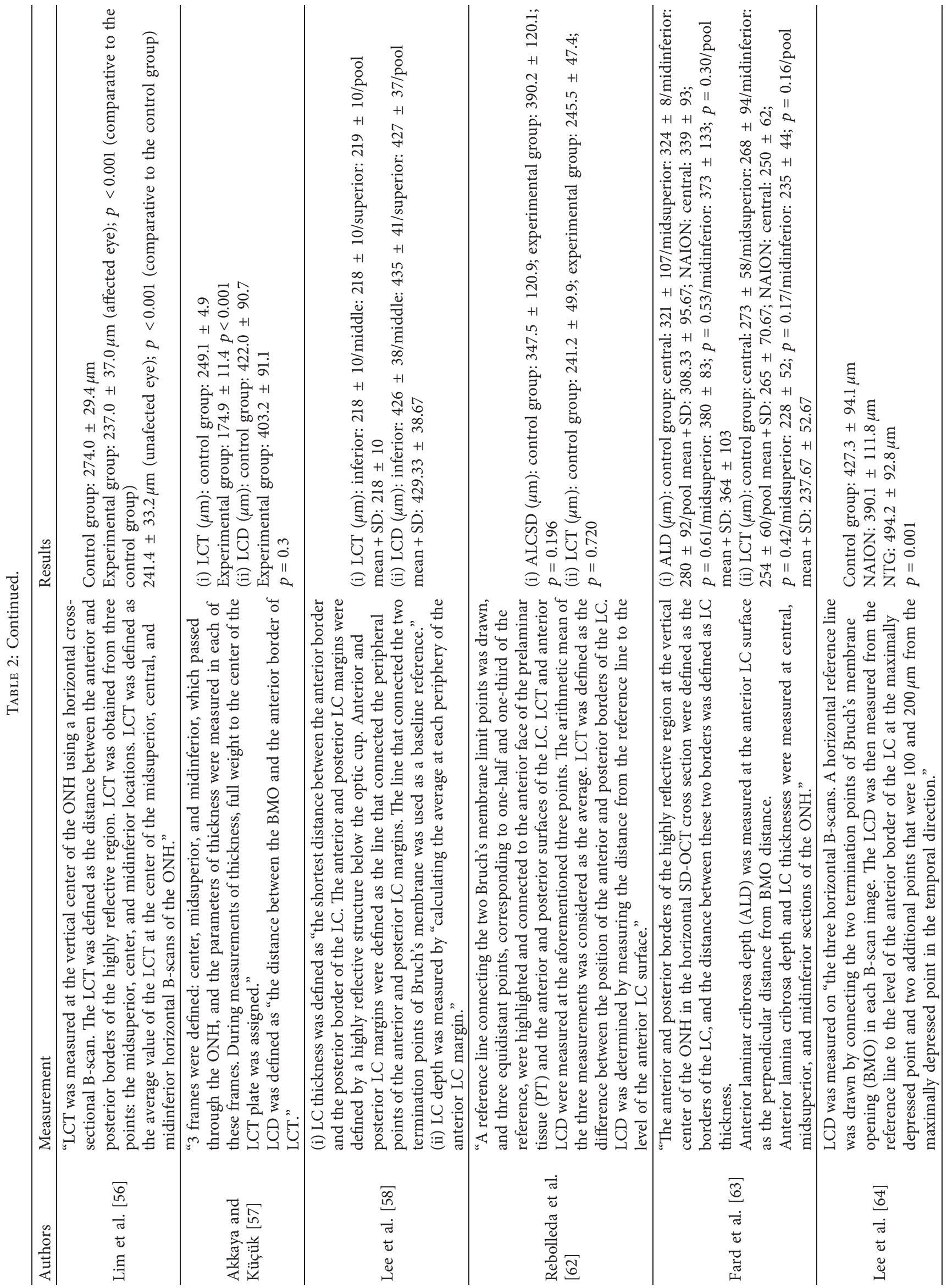



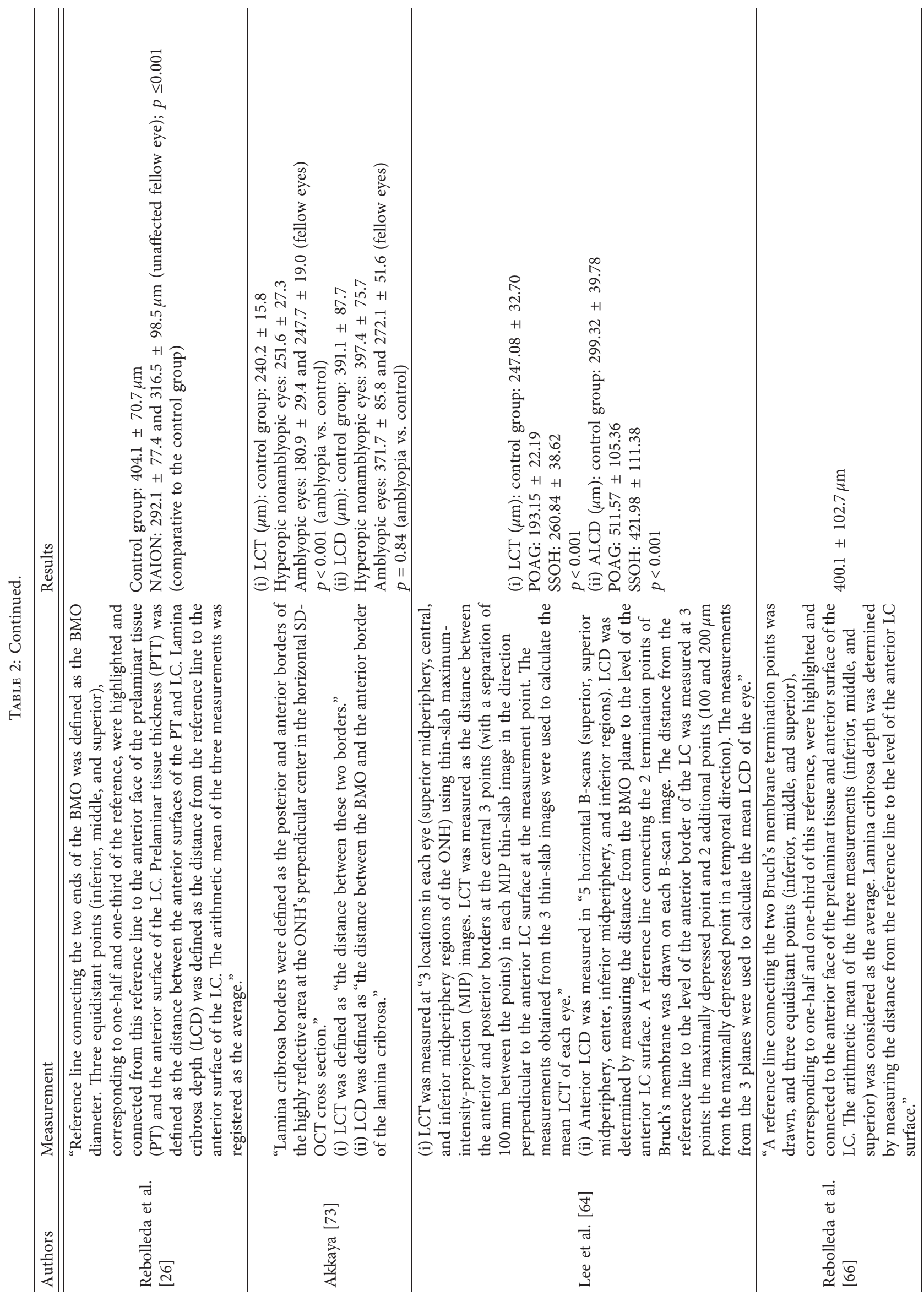


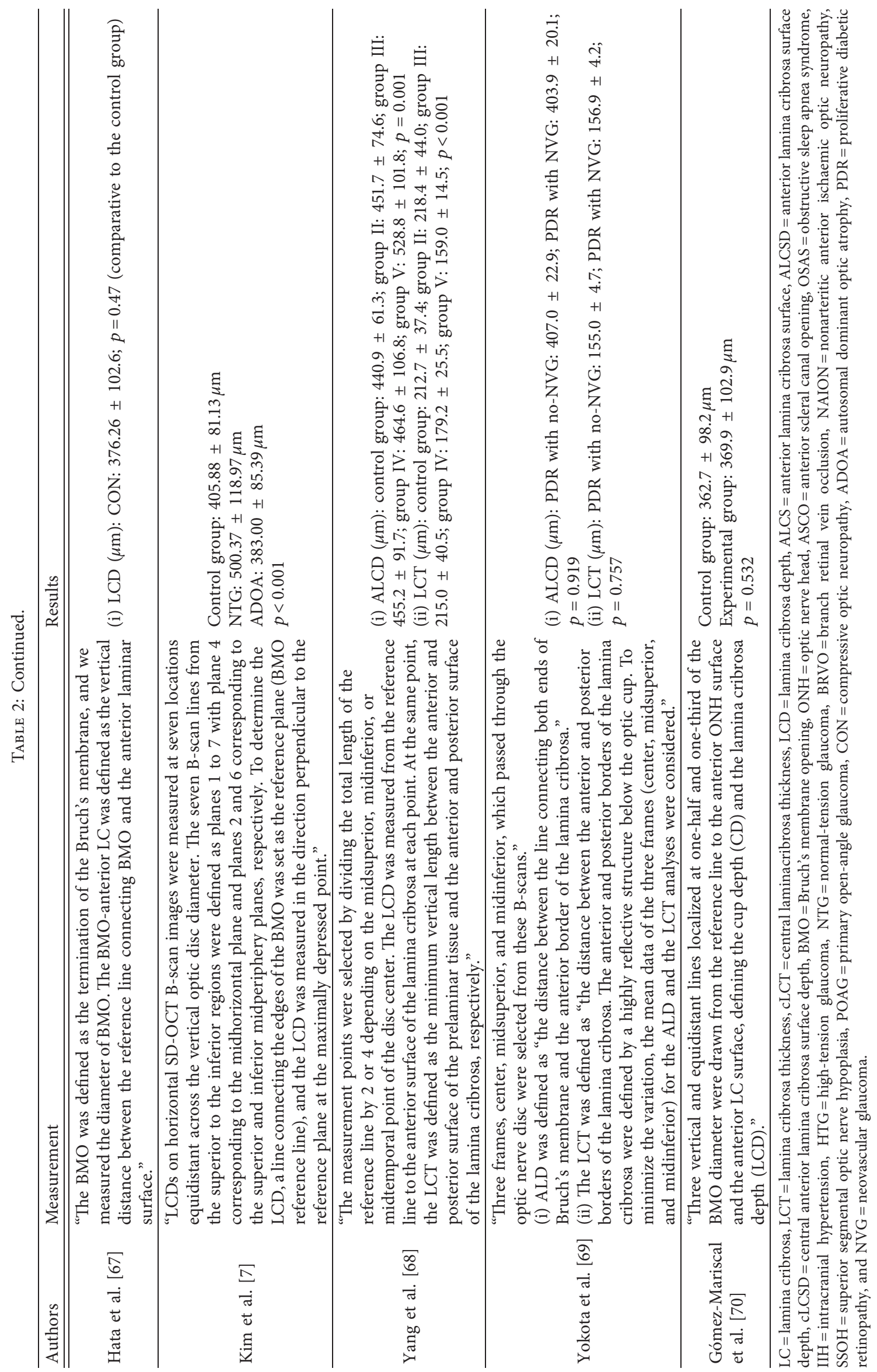




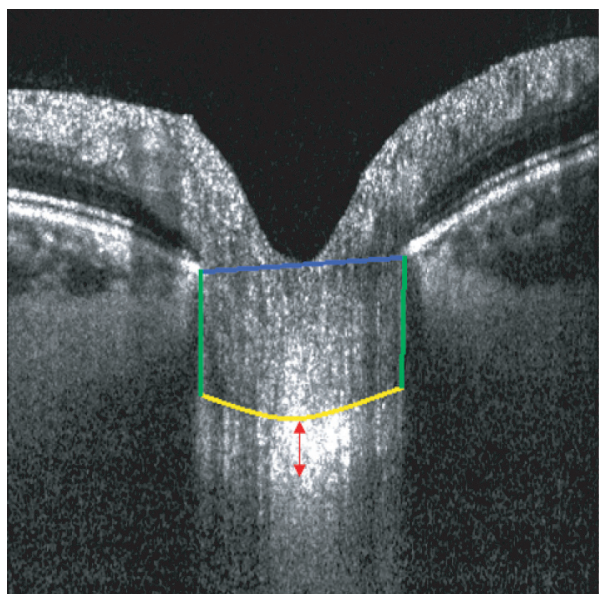

FIGURE 4: OCT B-scan showing the points used to compute the LCD. Line D in blue connects the two Bruch's membrane opening (BMO) edges. The two lines perpendicular to D in green are defined from the BMO edges to the anterior LC insertion points. The anterior LC surface is demarcated in yellow, enclosing the area $\mathrm{S}$ where the LC is measured. The red arrow measures the distance between the anterior and posterior margins of the LC, defined as the LCT.

significant because the standard deviations of all the diseases cross the standard deviation of the healthy LCD (Figure 6(b)). For example, Rebolleda et al. [26] found a lower average LCD in the superior, middle, and inferior planes in eyes with NAION compared to healthy eyes $(p<0.001)$, which was also true between unaffected fellow eyes when compared to healthy eyes $(p<0.05)$. The maximum value for LCD was found in cases of Graves' orbitopathy with proptosis and/or compressive optic neuropathy. Seo et al. [27] conducted a study in these patients and reported $462.79 \pm 95.96 \mu \mathrm{m}$ and $621.39 \pm 78.39 \mu \mathrm{m}$ values, at baseline, for the muscle-dominant and fat-dominant group, respectively.

3.3.3. Nonophthalmic Disease Group Measurements. The number of studies in this group was smaller than in the other groups. The registered diseases were diabetes mellitus [28], Parkinson's disease (PD) [29], obstructive sleep apnea syndrome (OSAS) [30], Alzheimer's disease (AD) [31, 32], mild cognitive impairment (MCI) [31], and migraine [33]. The mean LCT and LCD were $234 \pm 36 \mu \mathrm{m}$ and $390 \pm 68 \mu \mathrm{m}$, respectively, as shown in Figure 5. The graphics in Figure 7 presents LCT and LCD for each pathology in comparison to the healthy population (horizontal dashed green line).

LCT measurements seem to be lower relative to the healthy group, with the exception of diabetes mellitus. Akkaya et al. [28] described a significantly higher mean LCT in diabetic patients when compared to a healthy group, $271.61 \pm 33.96 \mu \mathrm{m}$ vs. $248.50 \pm 5.40 \mu \mathrm{m}$, respectively $(p<0.001)$. Regarding LCD, diabetes mellitus showed significantly lower mean values in comparison to healthy controls in one study [28] (351 $\pm 59 \mu \mathrm{m}$ vs. $420 \pm 90 \mu \mathrm{m}$; $p=0.003$ ). The maximum mean LCD absolute value (deepest ONH cup) was described in patients with migraine (see Figure 7(b)). Sirakaya et al. [33] reported significantly higher mean LCD values for both migraine groups
$(412.15 \pm 58.80 \mu \mathrm{m}$ with aura and $405.57 \pm 55.39 \mu \mathrm{m}$ without aura) when compared to the healthy group (355.34 $\pm 65.53 \mu \mathrm{m} ; p=0.001)$.

\section{Discussion}

The present study highlights which LC structural parameters have been analyzed in the literature with a focus on nonglaucomatous diseases. Overall, the most commonly studied parameters were LCD and LCT. The disease groups (ophthalmic and nonophthalmic) presented lower values for mean LCT, relative to the healthy population (Figure 5(a)). In parallel, mean LCD values were higher (deeper ONH cup) for these groups (Figure 5(b)). An exception in the nonophthalmic disease group was DM, which presented a shallower cup and thicker LC, when compared to healthy subjects. Akkaya et al. proposed that this evidence supports the "neuroprotective effect of DM on glaucomatous optic neuropathy and suggests that LCT and lamina cribrosa position mediate this protective effect." [28]

This study shows that LC structural features are significantly different between healthy patients and some (nonglaucomatous) ocular and systemic pathologies. As such, there is a potential to add them as additional clinical features for clinical diagnosis. Nonetheless, being patientspecific features, LC features might hold an even better role for patient follow-up, signalling disease status' change. Unfortunately, we did not find any longitudinal studies focusing on this matter in this review. This fact highlights the need for longitudinal studies linking LC parameters and diseases, similarly to what is now common in glaucomarelated studies [75].

LC features are influenced by factors such as age, race, and also by the way measurements are carried out. However, these factors were not used as segmentation criteria in this study due to the lack of this information in several studies. For future works in this field, it is important to take into account these factors when analysing 


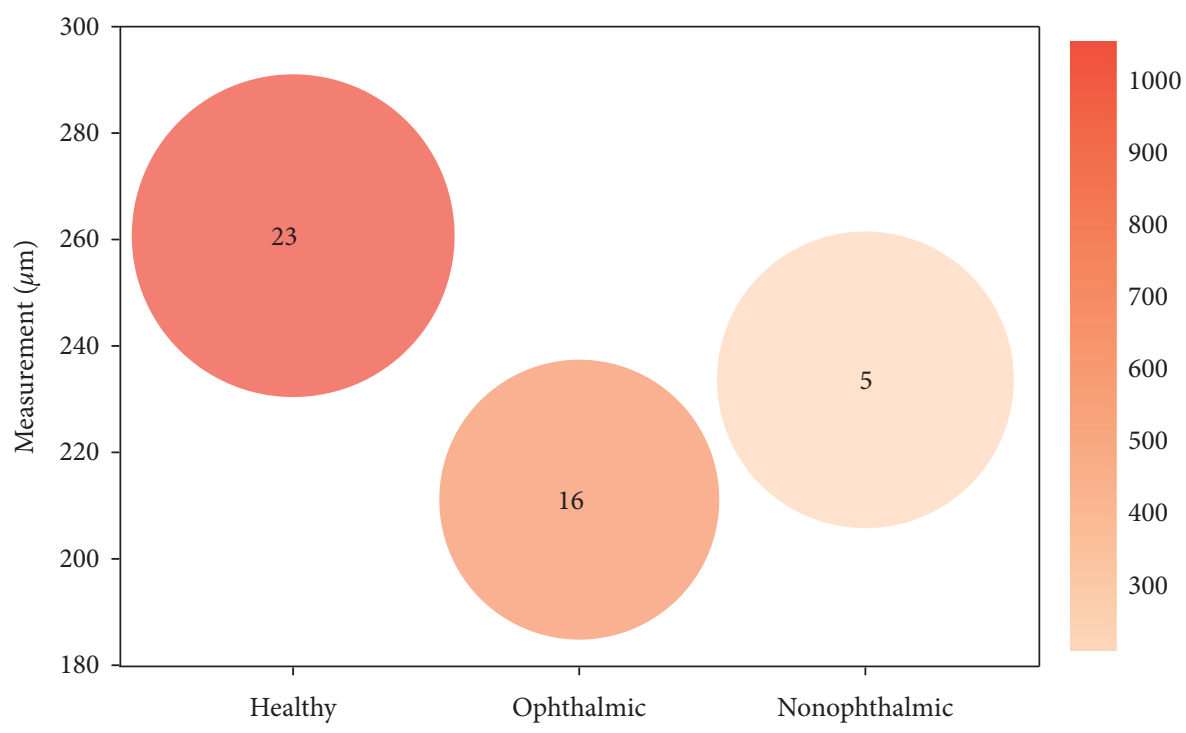

(a)

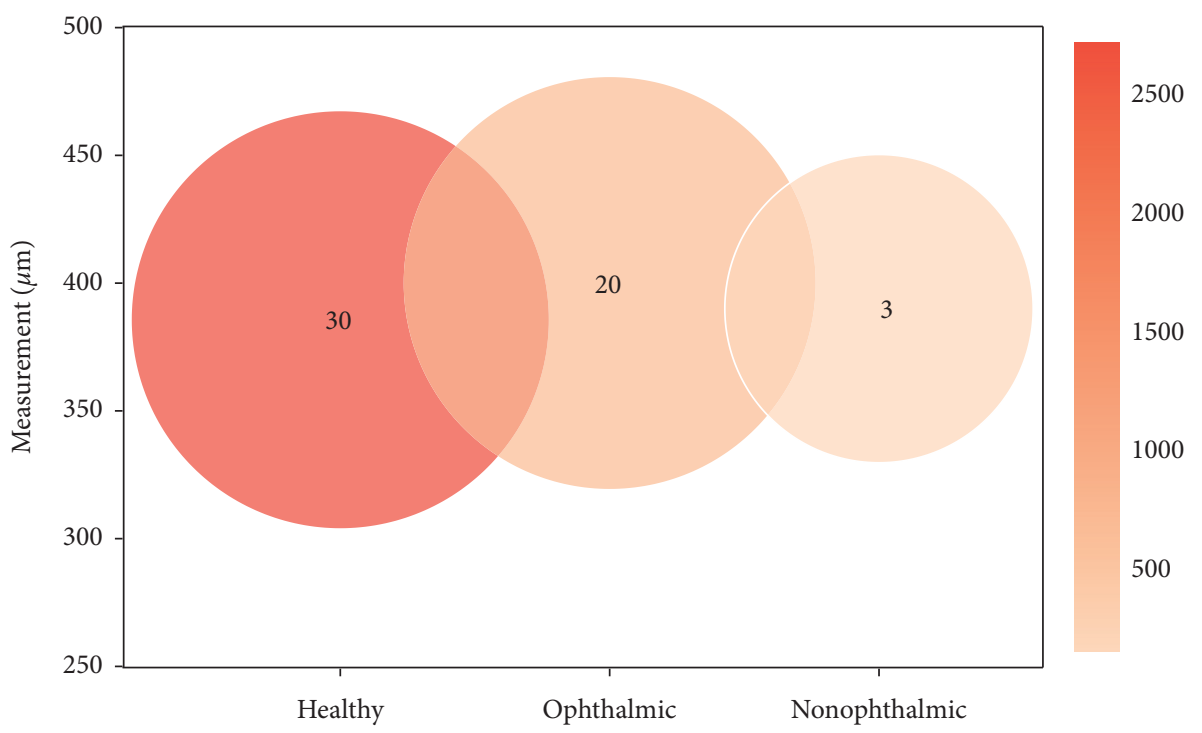

(b)

Figure 5: Comparison of lamina cribrosa (a) thickness (LCT) and (b) depth (LCD) between healthy controls, ophthalmic, and nonophthalmic diseases. The number in the circle represents the amount of studies used for calculating the averaged measurements for each group. The color scale shows the number of eyes comprised in the studied groups, and the radius of each circle denotes the standard deviation of the averaged values.

and comparing results between studies since they are a potential source of bias. Moreover, the current methods are heterogeneous (see Table 2), which may lead to imprecise comparisons between studies. For depth measurements, the consensus is to use the BMO plane as a reference, but the way the feature is measured is not consistent among research groups. One of the causes for this heterogeneity is the fact that the analysis of LC features still requires a considerable amount of manual input. This causes measurement bias due to the inherent difficulty of the manual delineation of the structure. This lack of automation increases the likelihood that each research group adopts their own reference points and methods. Besides, studies usually report averages of a limited number of Bscans without capturing the whole LC. The distance between these B-scan slices, as well as their number and position, may also be a source of discrepancies when comparing studies. Finally, some authors have pointed out the fact that the BMO reference place might be biased due to choroidal thickness changes and that perhaps the anterior sclera reference plane would be better suited for these calculations [76, 77]. Ideally, similar measurement methods should be adopted across all research groups. Currently, the measurement of the LC features is laborious and time consuming. As such, automation might hold the key to reduce bias in LC feature measurement. There is 


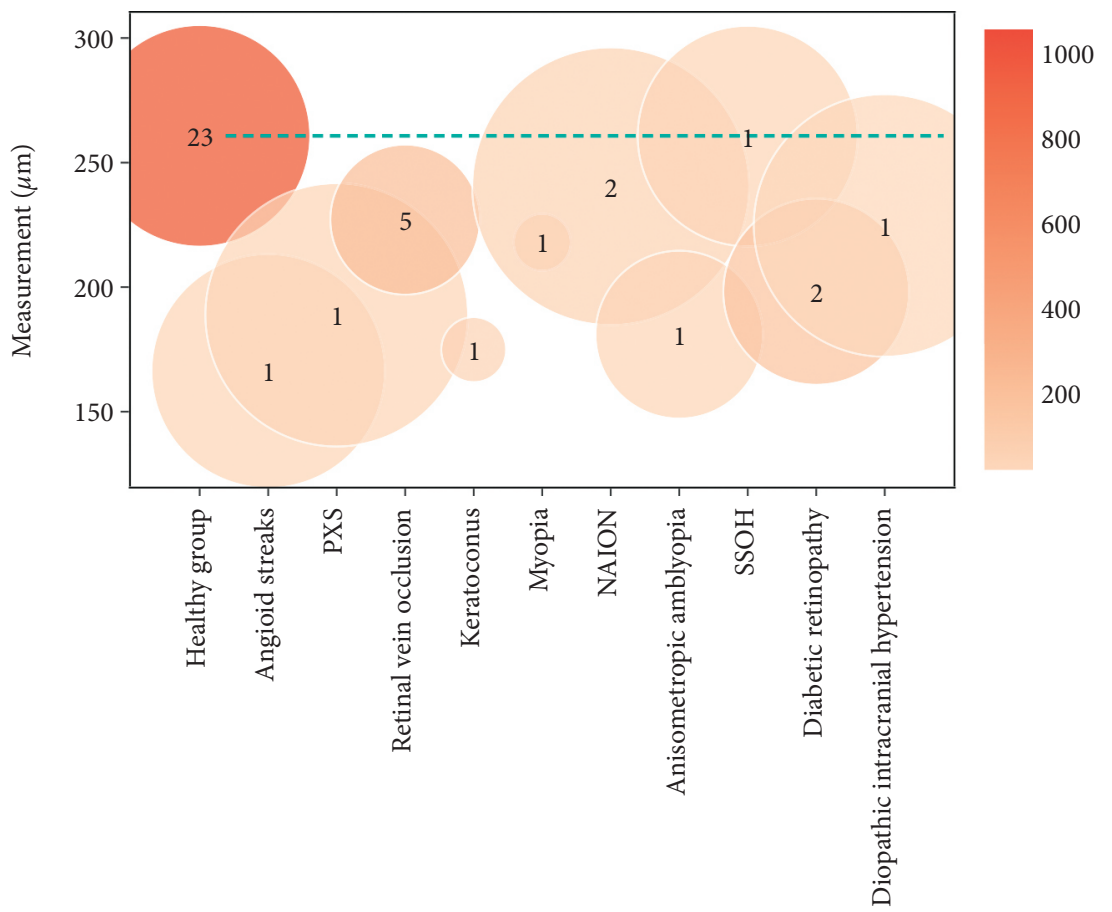

(a)

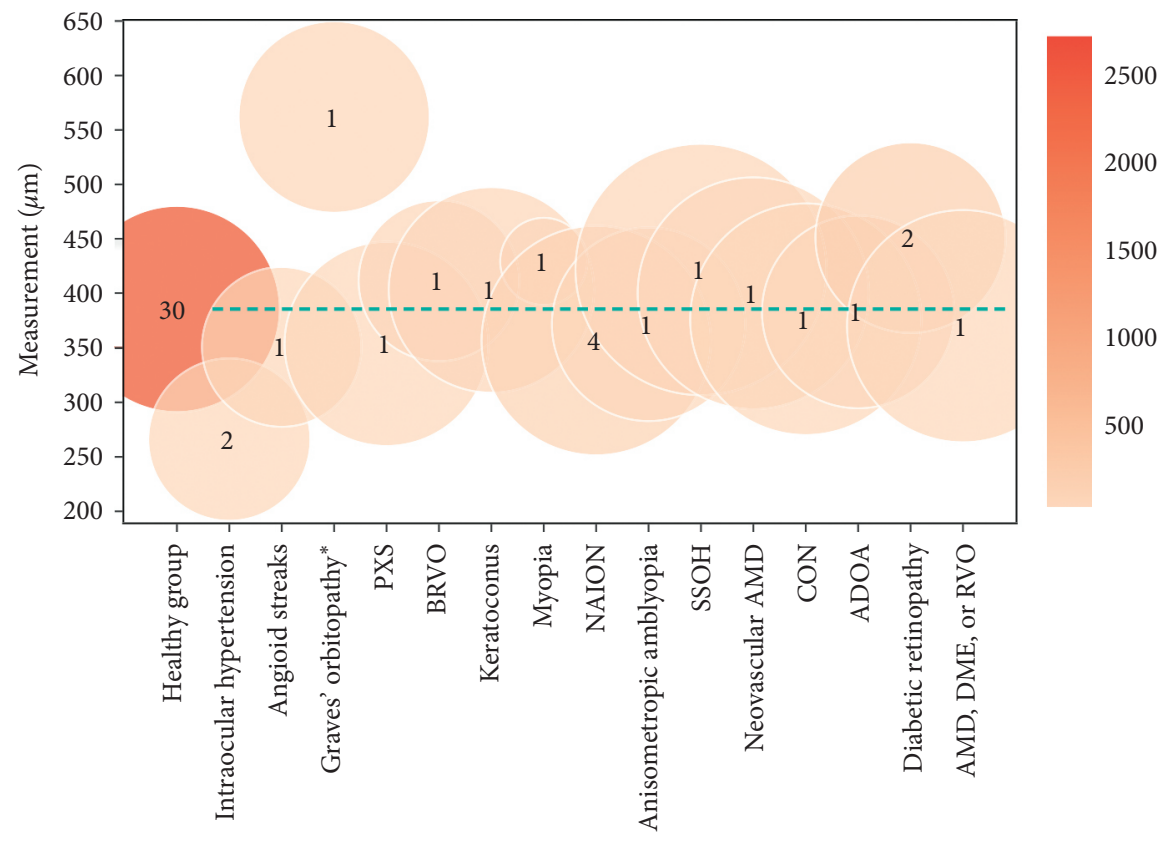

(b)

FIGURE 6: Comparison of lamina cribrosa (a) thickness (LCT) and (b) depth (LCD) for the ophthalmic disease group. The dashed green line represents the mean for the healthy population. Only pathologies that included more than 20 eyes among all articles are considered for the analysis. ${ }^{*}$ With proptosis and/or compressive optic neuropathy.

a need for easy-to-use software that can automatically measure LC features (possibly starting with LCT and LCD), ideally capturing all the information from OCT volumes, instead of selecting some of the B-scans. Providing such a tool with a fast and repeatable computation would contribute to making LC features a part of everyday clinical practice.
The main limitation of this review is the reduced number of studies, mainly in the nonophthalmic disease group, which precludes definite conclusions. Moreover, due to the lack of individual study data, it was not possible to perform statistical comparisons between groups and pathologies. As such, our results point towards differences that need to be better clarified. Nonetheless, LC features' 


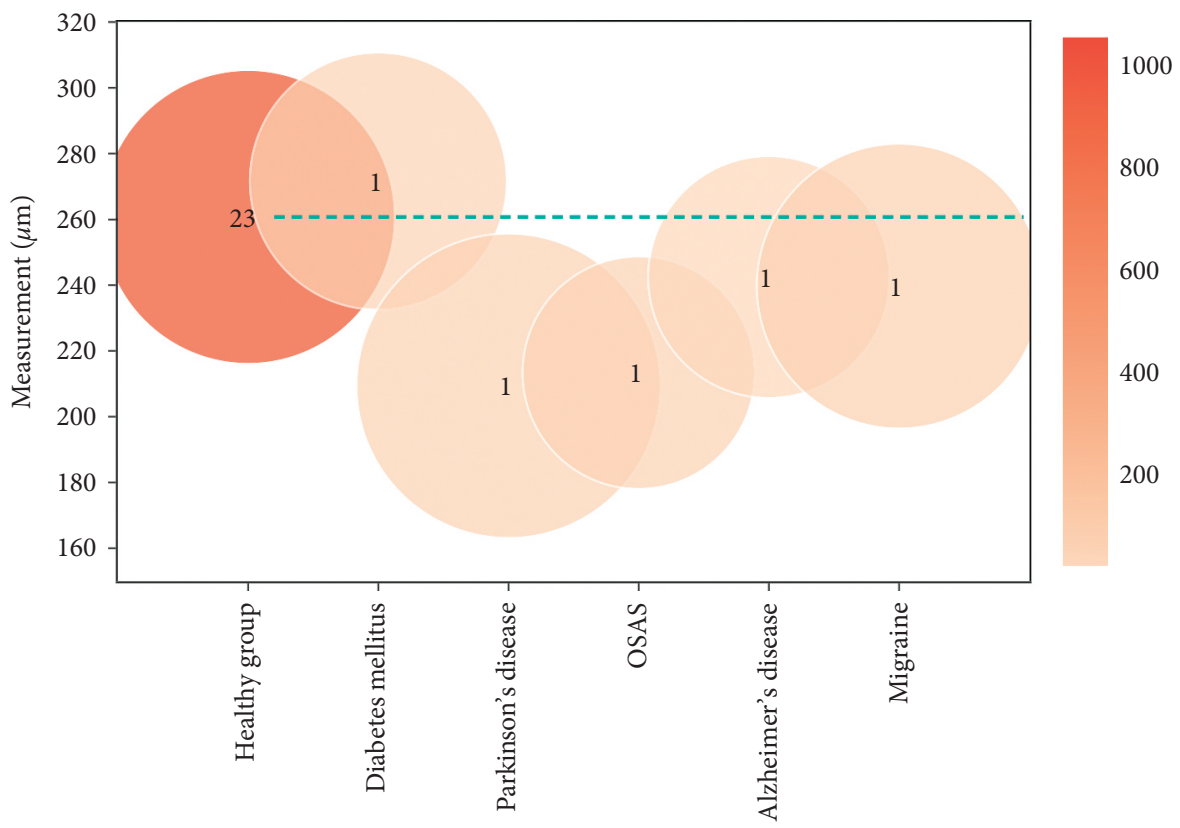

(a)

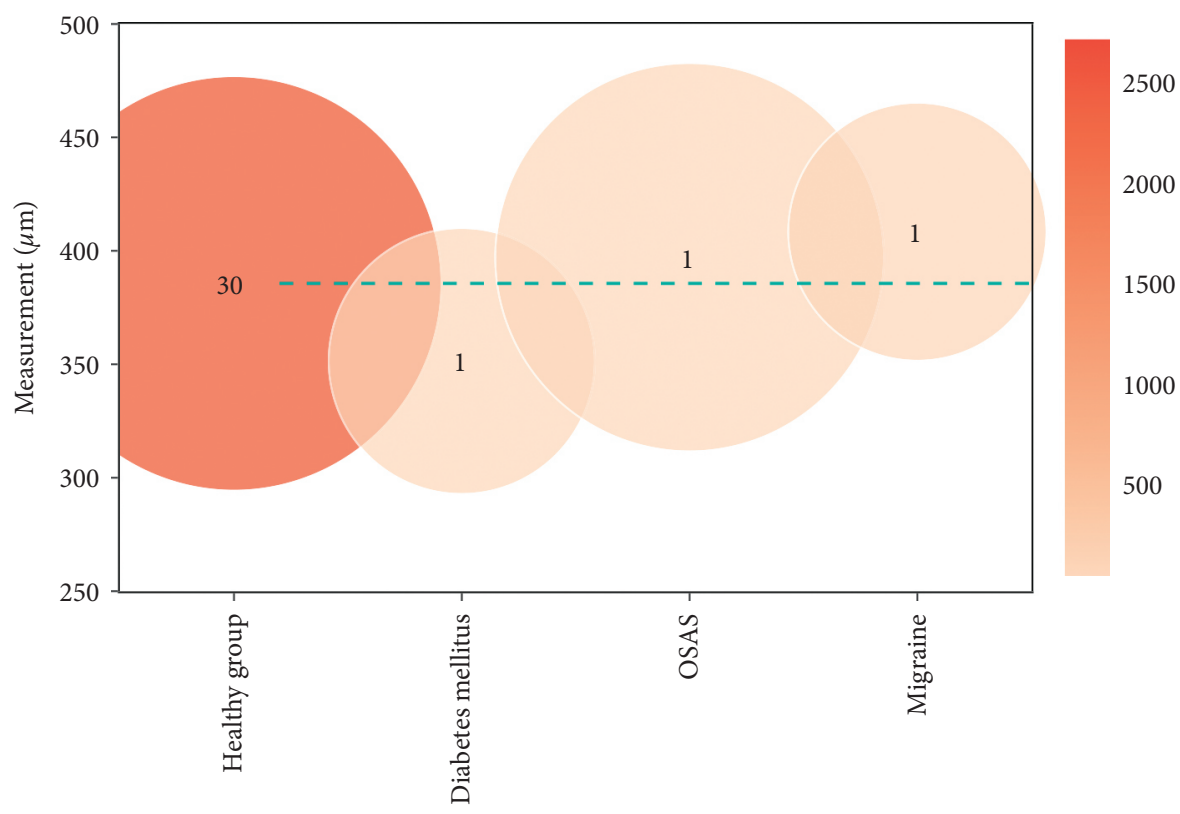

(b)

Figure 7: Comparison of lamina cribrosa (a) thickness (LCT) and (b) depth (LCD) for the nonophthalmic disease group. The green dashed line represents the mean for the healthy population. Only pathologies that included more than 20 eyes among all articles are considered for the analysis.

ability to discriminate between these groups is supported by results presented by several individual studies, as reported in Table 2. Lastly, it is noteworthy to mention that the statistical analysis performed on groups (ophthalmic and nonophthalmic diseases) may be biased by different pathologies comprised in each group.

\section{Conclusion}

There is a growing interest in LC features outside the glaucoma field. The results of this meta-analysis show several promising features (mainly, LCT and LCD) that may be relevant for clinical practice. Nevertheless, further studies are needed to validate these findings, and longitudinal data are needed to clarify the potential for use in patient follow-up. Moreover, efforts should be employed to develop automated tools that can capture LC features from OCT data in a standardized manner, thus allowing more accurate comparisons between studies. These efforts should enable to further explore the potential of LC parameters for use in daily clinical practice. 


\section{Conflicts of Interest}

The authors declare that they have no conflicts of interest.

\section{Acknowledgments}

This work was supported by the Horizon 2020 Research and Innovation Programme (grant agreement no. 780989: Multimodal, multi-scale retinal imaging project) and was funded by Portuguese National Funds through the FCT, Fundação Para a Ciência e a Tecnologia, I.P., in the scope of the project UIDB/04559/2020.

\section{References}

[1] I. A. Sigal, H. Yang, M. D. Roberts et al., "IOP-induced lamina cribrosa deformation and scleral canal expansion: independent or related?" Investigative Opthalmology \& Visual Science, vol. 52, no. 12, pp. 9023-9032, 2011.

[2] R. Grytz, M. A. Fazio, V. Libertiaux et al., "Age- and racerelated differences in human scleral material properties," Investigative Ophthalmology \& Visual Science, vol. 55, no. 12, pp. 8163-8172, 2014.

[3] H. Quigley and D. R. Anderson, "The dynamics and location of axonal transport blockade by acute intraocular pressure elevation in primate optic nerve," Investigative Ophthalmology, vol. 15, no. 8, pp. 606-616, 1976.

[4] H. A. Quigley, E. M. Addicks, W. R. Green, and A. E. Maumenee, "Optic nerve damage in human glaucoma. the site of injury and susceptibility to damage," Archives of Ophthalmology, vol. 99, no. 4, pp. 635-649, 1981.

[5] S. H. Lee, T.-W. Kim, E. J. Lee, M. J. A. Girard, and J. M. Mari, "Diagnostic power of lamina cribrosa depth and curvature in glaucoma," Investigative Opthalmology \& Visual Science, vol. 58, no. 2, pp. 755-762, 2017.

[6] X. Wang, T. A. Tun, M. E. Nongpiur et al., "Peripapillary sclera exhibits a v-shaped configuration that is more pronounced in glaucoma eyes," British Journal of Ophthalmology, 2020, In press.

[7] G.-N. Kim, J.-A. Kim, M.-J. Kim et al., "Comparison of lamina cribrosa morphology in normal tension glaucoma and autosomal-dominant optic atrophy," Investigative Ophthalmology \& Visual Science, vol. 61, no. 5, p. 9, 2020.

[8] J. C. Han, S. H. Cho, D. Y. Sohn, and C. Kee, "The characteristics of lamina cribrosa defects in myopic eyes with and without open-angle glaucoma," Investigative Opthalmology \& Visual Science, vol. 57, no. 2, pp. 486-494, 2016.

[9] D. S. Minckler and M. O. M. Tso, "A light microscopic, autoradiographic study of axoplasmic transport in the normal rhesus optic nerve head," American Journal of Ophthalmology, vol. 82, no. 1, pp. 1-15, 1976.

[10] A. Bhandari, L. Fontana, F. W. Fitzke, and R. A. Hitchings, "Quantitative analysis of the lamina cribrosa in vivo using a scanning laser opthalmoscope," Current Eye Research, vol. 16, no. 1, pp. 1-8, 1997.

[11] A. S. Vilupuru, N. V. Rangaswamy, L. J. Frishman, E. L. Smith III, R. S. Harwerth, and A. Roorda, "Adaptive optics scanning laser ophthalmoscopy for in vivo imaging of lamina cribrosa," Journal of the Optical Society of America A, vol. 24, no. 5, pp. 1417-1425, 2007.

[12] H.-Y. L. Park, S. H. Jeon, and C. K. Park, "Enhanced depth imaging detects lamina cribrosa thickness differences in normal tension glaucoma and primary open-angle glaucoma," Ophthalmology, vol. 119, no. 1, pp. 10-20, 2012.

[13] J. M. Mari, N. G. Strouthidis, S. C. Park, and M. J. A. Girard, "Enhancement of lamina cribrosa visibility in optical coherence tomography images using adaptive compensation," Investigative Opthalmology \& Visual Science, vol. 54, no. 3, pp. 2238-2247, 2013.

[14] B. Nuyen, K. Mansouri, and R. N. Weinreb, "Imaging of the lamina cribrosa using swept-source optical coherence tomography," Journal of Current Glaucoma Practice, vol. 6, no. 3, pp. 113-119, 2012.

[15] S. C. Park and R. Ritch, "High resolution in vivo imaging of the lamina cribrosa," Saudi Journal of Ophthalmology, vol. 25, no. 4, pp. 363-372, 2011.

[16] T.-W. Kim, L. Kagemann, M. J. A. Girard et al., "Imaging of the lamina cribrosa in glaucoma: perspectives of pathogenesis and clinical applications," Current Eye Research, vol. 38, no. 9, pp. 903-909, 2013.

[17] A. Bekkers, N. Borren, E. Vera et al., "Microvascular damage assessed by optical coherence tomography angiography for glaucoma diagnosis: a systematic review of the most discriminative regions," Acta Ophthalmologica, vol. 98, no. 6, pp. 537-558, 2020.

[18] J. C. Han, D.-Y. Choi, Y. K. Kwun, W. Suh, and C. Kee, "Evaluation of lamina cribrosa thickness and depth in ocular hypertension," Japanese Journal of Ophthalmology, vol. 60, no. 1, pp. 14-19, 2016.

[19] F. P. Bartolomé, J. M. Martínez de la Casa, I. C. Bosca et al., "Correlating corneal biomechanics and ocular biometric properties with lamina cribrosa measurements in healthy subjects," Seminars in Ophthalmology, vol. 33, no. 2, pp. $223-230,2018$.

[20] H. Xiao, X.-Y. Xu, Y.-M. Zhong, and X. Liu, "Age related changes of the central lamina cribrosa thickness, depth and prelaminar tissue in healthy Chinese subjects," International Journal of Ophthalmology, vol. 11, no. 11, pp. 1842-1847, 2018.

[21] L. A. Rhodes, C. Huisingh, J. Johnstone et al., "Variation of laminar depth in normal eyes with age and race," Investigative Ophthalmology \& Visual Science, vol. 55, no. 12, pp. 81238133, 2014.

[22] H. Luo, H. Yang, S. K. Gardiner et al., "Factors influencing central lamina cribrosa depth: a multicenter study," Investigative Opthalmology \& Visual Science, vol. 59, no. 6, pp. 2357-2370, 2018.

[23] S. C. Park, S. Kiumehr, C. C. Teng et al., "Horizontal central ridge of the lamina cribrosa and regional differences in laminar insertion in healthy subjects," Investigative Ophthalmology \& Visual Science, vol. 53, no. 3, pp. 1610-1616, 2012.

[24] D. S. Lee, E. J. Lee, T.-W. Kim et al., "Influence of translaminar pressure dynamics on the position of the anterior lamina cribrosa surface," Investigative Opthalmology \& Visual Science, vol. 56, no. 5, pp. 2833-2841, 2015.

[25] J. M. Villarruel, X. Q. Li, D. Bach-Holm, and S. Hamann, "Anterior lamina cribrosa surface position in idiopathic intracranial hypertension and glaucoma," European Journal of Ophthalmology, vol. 27, no. 1, pp. 55-61, 2017.

[26] G. Rebolleda, A. Pérez-Sarriegui, L. Díez-Álvarez et al., "Lamina cribrosa position and bruch's membrane opening differences between anterior ischemic optic neuropathy and open-angle glaucoma," European Journal of Ophthalmology, vol. 29, no. 2, pp. 202-209, 2018.

[27] Y. Seo, W. B. Shin, H. W. Bae, and J. S. Yoon, "Effects of orbital decompression on lamina cribrosa depth in patients 
with Graves' orbitopathy," Korean Journal of Ophthalmology, vol. 33, no. 5, pp. 436-445, 2019.

[28] S. Akkaya, B. Küçük, H. K. Doğan, and E. Can, "Evaluation of the lamina cribrosa in patients with diabetes mellitus using enhanced depth imaging spectral-domain optical coherence tomography," Diabetes and Vascular Disease Research, vol. 15, no. 5, pp. 442-448, 2018.

[29] M. Eraslan, C. Eren, S. Yildiz Balci et al., "The choroid and lamina cribrosa is affected in patients with Parkinson's disease: enhanced depth imaging optical coherence tomography study," Acta Ophthalmologica, vol. 94, no. 1, pp. 68-75, 2016.

[30] B. Küçük, E. Sırakaya, and Ş Delibaş, "Posterior segment assessment in patients with obstructive sleep apnea syndrome," Sleep \& Breathing, vol. 23, no. 3, pp. 997-1005, 2019.

[31] A. López-de Eguileta, C. Lage, S. López-García et al., "Ganglion cell layer thinning in prodromal Alzheimer's disease defined by amyloid pet," Alzheimer's \& Dementia, vol. 5, pp. 570-578, 2019.

[32] E. J. Lee, T.-W. Kim, D. S. Lee et al., "Increased CSF tau level is correlated with decreased lamina cribrosa thickness," Alzheimer's Research \& Therapy, vol. 8, no. 6, 2016.

[33] E. Sirakaya, B. Kucuk, A. Agadayi, and N. Yilmaz, "Evaluation of the lamina cribrosa thickness and depth in patients with migraine," International Ophthalmology, vol. 40, no. 1, pp. 89-98, 2020.

[34] D. C. Sousa, I. Leal, C. Marques-Neves, F. Pinto, and L. Abegão Pinto, "Relationship between intraocular pressure and anterior lamina cribrosa depth: a cross-sectional observational study in a healthy Portuguese population," European Journal of Ophthalmology, vol. 27, no. 3, pp. 295-300, 2017.

[35] E. J. Lee, T.-W. Kim, R. N. Weinreb, M. H. Suh, and H. Kim, "Lamina cribrosa thickness is not correlated with central corneal thickness or axial length in healthy eyes: central corneal thickness, axial length, and lamina cribrosa thickness," Graefe's Archive for Clinical and Experimental Ophthalmology, vol. 251, no. 3, pp. 847-854, 2013.

[36] I. Leal, D. C. Sousa, F. Pinto, C. Marques-Neves, and L. Abegão Pinto, "Intra- and inter-rater agreement of anterior lamina cribrosa depth measurements using enhanced-depth imaging optical coherence tomography," Ophthalmic Research, vol. 57, no. 2, pp. 92-99, 2017.

[37] X. Wang, M. R. Beotra, T. A. Tun et al., "In vivo 3-dimensional strain mapping confirms large optic nerve head deformations following horizontal eye movements," Investigative Opthalmology \& Visual Science, vol. 57, no. 13, pp. 5825-5833, 2016.

[38] Y. W. Kim, M. J. A. Girard, J. M. Mari, and J. W. Jeoung, "Anterior displacement of lamina cribrosa during valsalva maneuver in young healthy eyes," PLoS One, vol. 11, no. 7, Article ID e0159663, 2016.

[39] M. Poli, P. Denis, E. Sellem, L.-S. Aho-Glélé, and A. M. Bron, "Is the optic nerve head structure impacted by a diagnostic lumbar puncture in humans?" Journal of Glaucoma, vol. 26, no. 11, pp. 1036-1040, 2017.

[40] A. El-Agamy, F. Oteaf, and M. Berika, "Anterior lamina cribrosa surface depth in healthy saudi females," Clinical Ophthalmology, vol. 11, pp. 1045-1050, 2017.

[41] P. Bedggood, F. Tanabe, A. M. McKendrick, A. Turpin, A. J. Anderson, and B. V. Bui, "Optic nerve tissue displacement during mild intraocular pressure elevation: its relationship to central corneal thickness and corneal hysteresis," Ophthalmic and Physiological Optics, vol. 38, no. 4, pp. 389399, 2018.
[42] S. H. Lee, T.-W. Kim, E. J. Lee et al., "Lamina cribrosa curvature in healthy Korean eyes," Scientific Reports, vol. 9, no. 1, p. 1756, 2019.

[43] J. H. Seo, T.-W. Kim, and R. N. Weinreb, "Lamina cribrosa depth in healthy eyes," Investigative Opthalmology \& Visual Science, vol. 55, no. 3, pp. 1241-1251, 2014.

[44] M. A. Fazio, J. K. Johnstone, B. Smith, L. Wang, and C. A. Girkin, "Displacement of the lamina cribrosa in response to acute intraocular pressure elevation in normal individuals of African and European descent," Investigative Opthalmology \& Visual Science, vol. 57, no. 7, pp. 3331-3339, 2016.

[45] T. A. Tun, X. Wang, M. Baskaran et al., "Variation of peripapillary scleral shape with age," Investigative Opthalmology \& Visual Science, vol. 60, no. 10, pp. 3275-3282, 2019.

[46] S. G. Thakku, Y.-C. Tham, M. Baskaran et al., "A global shape index to characterize anterior lamina cribrosa morphology and its determinants in healthy indian eyes," Investigative Opthalmology \& Visual Science, vol. 56, no. 6, pp. 3604-3614, 2015.

[47] I. Pasaoglu, B. Satana, C. Altan et al., "Lamina cribrosa surface position in idiopathic intracranial hypertension with sweptsource optical coherence tomography," Indian Journal of Ophthalmology, vol. 67, no. 7, pp. 1085-1088, 2019.

[48] J. García-Montesinos, F. J. Muñoz-Negrete, V. de Juan, and G. Rebolleda, "Relationship between lamina cribrosa displacement and trans-laminar pressure difference in papilledema," Graefe's Archive for Clinical and Experimental Ophthalmology, vol. 255, no. 6, pp. 1237-1243, 2017.

[49] G. Demir, C. Altan, S. Cakmak et al., "Evaluation of lamina cribrosa in angioid streaks using spectral-domain optical coherence tomography enhanced depth imaging," Journal Français d'Ophtalmologie, vol. 42, no. 6, pp. 586-591, 2019.

[50] S. Moghimi, M. Mazloumi, M. Johari et al., "Evaluation of lamina cribrosa and choroid in nonglaucomatous patients with pseudoexfoliation syndrome using spectral-domain optical coherence tomography," Investigative Opthalmology \& Visual Science, vol. 57, no. 3, pp. 1293-1300, 2016.

[51] A. Soares, N. Lopes, G. Morgado et al., "Study of lamina cribrosa depth and optic nerve in patients with spontaneous intracranial hypotension," European Journal of Ophthalmology, vol. 29, no. 6, pp. 659-663, 2019.

[52] S. Karaca Adiyeke, N. Kutlu, H. Aytogan et al., "Thickness of sclera and lamina cribrosa in patients with central retinal vein occlusion," Retina, vol. 40, no. 10, pp. 2050-2054, 2019.

[53] Y. Son, S. Lee, and J. Park, "Measurement of lamina and prelaminar thicknesses of both eyes in patients with unilateral branch retinal vein occlusion," Graefe's Archive for Clinical and Experimental Ophthalmology, vol. 255, no. 3, pp. 503-508, 2017.

[54] E. Sirakaya and K. Bekir, "Thickness of the lamina cribrosa, retinal nerve fiber layer, and peripapillary choroid in patients with branch retinal vein occlusion," Ophthalmologica, vol. 243, no. 4, pp. 288-296, 2019.

[55] O. Altunel, M. Atas, and S. Demircan, "Evaluation of lamina cribrosa thickness in patients diagnosed with central retinal vein occlusion," Graefe's Archive for Clinical and Experimental Ophthalmology, vol. 257, no. 10, pp. 2087-2093, 2019.

[56] S.-H. Lim, M. Kim, W. Chang, and M. Sagong, "Comparison of the lamina cribrosa thickness of patients with unilateral branch retinal vein occlusion and healthy subjects," Retina, vol. 37, no. 3, pp. 515-521, 2017. 
[57] S. Akkaya and B. Küçük, "Lamina cribrosa thickness in patients with keratoconus," Cornea, vol. 36, no. 12, pp. 1509-1513, 2017.

[58] S. Lee, D.-Y. D. Choi, D. H. Lim, T. Y. Chung, J. C. Han, and K. C. Lim, "Lamina cribrosa changes after laser in situ keratomileusis in myopic eyes," Korean Journal of Ophthalmology, vol. 32, no. 2, pp. 95-102, 2018.

[59] A. Jnawali, H. Mirhajianmoghadam, G. Musial et al., "The optic nerve head, lamina cribrosa, and nerve fiber layer in non-myopic and myopic children," Experimental Eye Research, vol. 195, 2020.

[60] K. Ohno-Matsui, M. Akiba, M. Moriyama, T. Ishibashi, T. Tokoro, and R. F. Spaide, "Imaging retrobulbar subarachnoid space around optic nerve by swept-source optical coherence tomography in eyes with pathologic myopia," Investigative Opthalmology \& Visual Science, vol. 52, no. 13, pp. 9644-9650, 2011.

[61] A. Miki, Y. Ikuno, T. Asai, S. Usui, and K. Nishida, "Defects of the lamina cribrosa in high myopia and glaucoma," PLoS One, vol. 10, no. 9, Article ID e0137909, 2015.

[62] G. Rebolleda, J. García-Montesinos, E. De Dompablo, N. Oblanca, F. J. Muñoz-Negrete, and J. J. González-López, "Bruch's membrane opening changes and lamina cribrosa displacement in non-arteritic anterior ischaemic optic neuropathy," British Journal of Ophthalmology, vol. 101, no. 2, pp. 143-149, 2017.

[63] M. A. Fard, M. Afzali, P. Abdi et al., "Optic nerve head morphology in nonarteritic anterior ischemic optic neuropathy compared to open-angle glaucoma," Investigative Opthalmology \& Visual Science, vol. 57, no. 11, pp. 4632-4640, 2016.

[64] E. J. Lee, Y. J. Choi, T.-W. Kim, and J.-M. Hwang, "Comparison of the deep optic nerve head structure between normal-tension glaucoma and nonarteritic anterior ischemic optic neuropathy," PLoS One, vol. 11, no. 4, Article ID e0150242, 2016.

[65] K. Shinohara, M. Moriyama, N. Shimada et al., "Analyses of shape of eyes and structure of optic nerves in eyes with tilted disc syndrome by swept-source optical coherence tomography and three-dimensional magnetic resonance imaging," Eye, vol. 27, no. 11, pp. 1233-1242, 2013.

[66] G. Rebolleda, B. Puerto, V. de Juan, M. Gómez-Mariscal, F. J. Muñoz-Negrete, and A. Casado, "Optic nerve head biomechanic and IOP changes before and after the injection of aflibercept for neovascular age-related macular degeneration," Investigative Opthalmology \& Visual Science, vol. 57, no. 13, pp. 5688-5695, 2016.

[67] M. Hata, K. Miyamoto, A. Oishi et al., "Comparison of optic disc morphology of optic nerve atrophy between compressive optic neuropathy and glaucomatous optic neuropathy," PLoS One, vol. 9, no. 11, Article ID e0112403, 2014.

[68] H. S. Yang, J.-G. Kim, J. B. Cha et al., "Quantitative analysis of neural tissues around the optic disc after panretinal photocoagulation in patients with diabetic retinopathy," PLoS One, vol. 12, no. 10, Article ID e0186229, 2017.

[69] S. Yokota, Y. Takihara, Y. Takamura, and M. Inatani, "Circumpapillary retinal nerve fiber layer thickness, anterior lamina cribrosa depth, and lamina cribrosa thickness in neovascular glaucoma secondary to proliferative diabetic retinopathy: a cross-sectional study," BMC Ophthalmology, vol. 17, no. 1, p. 57, 2017.

[70] M. Gómez-Mariscal, B. Puerto, F. J. Muñoz-Negrete, V. de Juan, and G. Rebolleda, "Acute and chronic optic nerve head biomechanics and intraocular pressure changes in patients receiving multiple intravitreal injections of antivegf," Graefe's Archive for Clinical and Experimental Ophthalmology, vol. 257, no. 10, pp. 2221-2231, 2019.

[71] D. F. Garway-Heath, D. Poinoosawmy, F. W. Fitzke, and R. A. Hitchings, "Mapping the visual field to the optic disc in normal tension glaucoma eyes," Ophthalmology, vol. 107, no. 10, pp. 1809-1815, 2000.

[72] T. Li, I. J. Saldanha, J. Jap et al., “A randomized trial provided new evidence on the accuracy and efficiency of traditional vs. electronically annotated abstraction approaches in systematic reviews," Journal of Clinical Epidemiology, vol. 115, pp. 77-89, 2019.

[73] S. Akkaya, "Lamina cribrosa thickness in children with hyperopic anisometropic amblyopia," International Journal of Ophthalmology, vol. 11, no. 10, pp. 1663-1667, 2018.

[74] E. J. Lee, T.-W. Kim, and R. N. Weinreb, "Improved reproducibility in measuring the laminar thickness on enhanced depth imaging sd-oct images using maximum intensity projection," Investigative Opthalmology \& Visual Science, vol. 53, no. 12, pp. 7576-7582, 2012.

[75] N. Y. Q. Tan, Y.-C. Tham, S. G. Thakku et al., "Changes in the anterior lamina cribrosa morphology with glaucoma severity," Scientific Reports, vol. 9, no. 6612, 2019.

[76] H. Quigley, K. Arora, S. Idrees et al., "Biomechanical responses of lamina cribrosa to intraocular pressure change assessed by optical coherence tomography in glaucoma eyes," Investigative Opthalmology \& Visual Science, vol. 58, no. 5, pp. 2566-2577, 2017.

[77] J. R. Vianna, V. R. Lanoe, J. Quach et al., "Serial changes in lamina cribrosa depth and neuroretinal parameters in glaucoma: impact of choroidal thickness," Ophthalmology, vol. 124, no. 9, pp. 1392-1402, 2017. 